\title{
Simulation Investigation of Bulk and Surface Properties of Liquid Benzonitrile: Ring Stacking - Assessment and Deconvolution
}

\author{
Leila Sakhtemanian, Mohammad Hadi Ghatee* \\ (Department of Chemistry, Shiraz University, Shiraz, 71946 Iran)
}

E-mail: mhghatee@shirazu.ac.ir mhghatee2@gmail.com

Fax: +98 7136460788, Tel: +98 7136137174

*Corresponding Author 


\begin{abstract}
This manuscript is devoted to classical molecular dynamics (MD) simulation studies of the bulk and surface properties of liquid benzonitrile (BZN) in the temperature range of 293-323K. The content and the simulation-analysis are inspired by our recent ab initio calculation on benzonitrile, whereas present results are to expand and develop macroscopic documentation involving data verification. We investigate the molecular stacking that involves phenyl ring, which is notably absent in the counterpart acetonitrile solvent. MD simulations of the bulk liquid unravel the hydrogen bond $(-\mathrm{C} \equiv \mathrm{N} \cdots \mathrm{H})$ formation and strength, in the order of ortho-H > meta-H $\sim$ para-H. The possibility for ortho-H's to get involved in the formation of two bonds simultaneously confirms each having $\sigma$ - and $\pi$-bonding features. The singularity centered at about $313 \mathrm{~K}$ found in the trend of the simulated temperature-dependent viscosity and diffusion coefficient of liquid BZN goes alongside the reported experiment, and the phenomenon may root from a change in the internal frictional motion of the molecular cluster in stacking modes. Accordingly, we used vast efforts for analysis particularly based on the deconvolution of the corresponding complex correlation functions. Specific angle-dependent correlation functions led to the recognition of the stacking molecules and their strict orientational character by utilizing relative molecular twist angles. Recognition of the strict orientational character of the stacking molecules, as a clue to the singularity in the viscosity trend, will be discussed based on specific angle-dependent correlation functions.
\end{abstract}

Keywords: Angle-dependent correlation function; Benzonitrile stacking; Liquid bulk and surface; MD simulation; Singularity and deconvolution; Temperature-dependent viscosity and diffusion 


\section{Table of content}

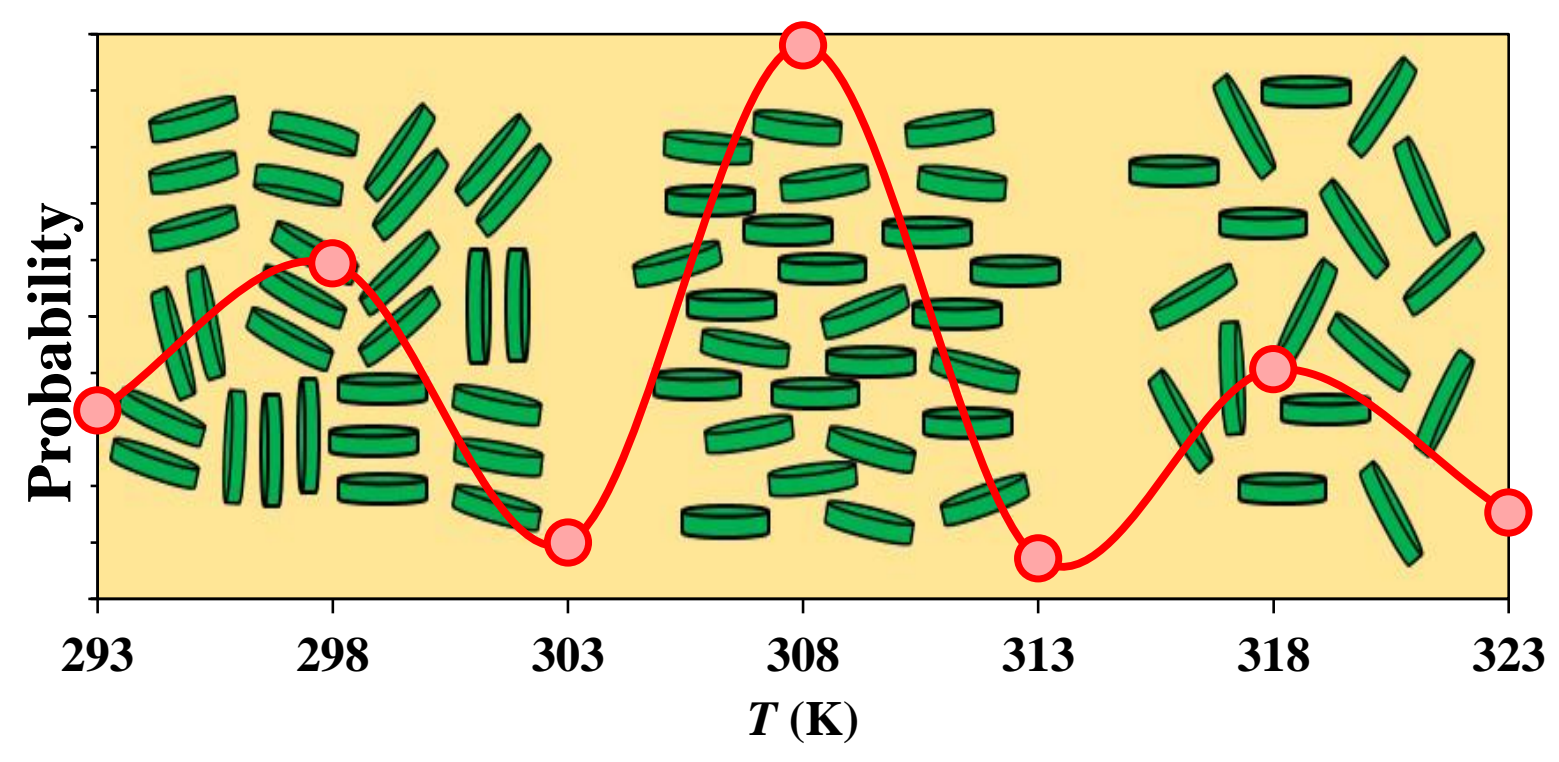




\section{INTRODUCTION}

As the simplest aromatic nitrile, benzonitrile $\left(\mathrm{C}_{6} \mathrm{H}_{5} \mathrm{CN}\right.$, cyanobenzene) provides a good bridging point between aliphatic nitriles, already extensively researched, and more complex, substituted species that have greater industrial relevance in several sectors of the chemical manufacturing industry such as pharmaceuticals and agrochemicals. Also, this molecule has a wide distribution of natural products. ${ }^{1}$ As the simplest nitrogen-bearing aromatic molecule, benzonitrile has been discovered recently in the interstellar medium. ${ }^{2,3}$ Benzonitrile and its derivatives are comprising an important class of organic compounds in the chemical industry. This is a stable compound to pyrolysis ${ }^{4}$ an essential process in the synthesis of benzoic acid, benzylamine, benzamide, pesticides, fatty amines, dyes and could be precursors for the synthesis of benzylamine, which can be used as a pharmaceutical intermediate. ${ }^{5,6}$

Also, benzonitrile is generally a good solvent for the synthesis of organic, anhydrous inorganic, and organometallic compounds and is generally similar in its behavior as a solvent to acetonitrile $\left(\mathrm{CH}_{3} \mathrm{CN}\right){ }^{7}$ But, benzonitrile differs from acetonitrile in two interesting and significant ways: it has delocalized $\pi$-electrons in the phenyl ring constantly interacting with the $-\mathrm{C} \equiv \mathrm{N}$ group, and it has a large phenyl group as compared to the methyl group creating a steric problem. ${ }^{8}$ Furthermore, the benzonitrile unlike acetonitrile have not a hydrogen atom in the alpha position to the nitrile group. While acetonitrile and benzonitrile have the same functional group $-\mathrm{C} \equiv \mathrm{N}$, the differences must be sought out in the contribution of the delocalized electrons of the phenyl ring in benzonitrile. More specifically, the cyano group in benzonitriles represents one of the most prevalent functional groups and can feasibly transfer to other functional moieties, such as aldehydes, carboxylic acids, amidines, amides, and amines. ${ }^{1}$ The cyano compounds show larger discrepancies relative to the analogous nitro compounds. This is an observable difference between benzonitrile and nitrobenzene even though the dipole moments are similar and the two compounds have equal polarizabilities. This 
may be explained in terms of a more localized charge in benzonitrile which results in a strongly oriented dipole-moment vector. ${ }^{9}$ Benzonitrile is an interesting molecule because of its ability to form a variety of hydrogen-bonded structures, having three different sites through which may form a hydrogen bond: (1) the $\pi$-electrons of the $\mathrm{CN}$ triple bond, (2) nonbonding electrons of the nitrogen atom of the $\mathrm{CN}$ group, and (3) the $\pi$-electrons of the phenyl ring. ${ }^{10}$ The features of these interactions have been characterized recently ${ }^{11}$ by studying the specification of benzonitrile cluster formation of different sizes by quantum chemical calculations.

Lots of (experimental, theoretical, and computational) research works have shown interest in benzonitrile and its derivatives, and recently, it has found other applications such as cosolvent because of large dipole moment $\left(4.01 \mathrm{D}^{12}\right.$ and $\left.4.18 \mathrm{D}^{13}\right)$ and widespread strong miscibility. ${ }^{14-16}$ Because of the three different binding sites, benzonitrile has shown interesting adsorption features on metal and non-metal surfaces. So far, various studies have been performed on the adsorption of benzonitrile on the $\mathrm{Ag}, \mathrm{Ni}, \mathrm{Pd}, \mathrm{Pt}, \mathrm{Au}$ and, Fe-doped carbon nanostructures surfaces. ${ }^{17-22}$ Also, the structure of various benzonitrile clusters was investigated through different theoretical ${ }^{11}$ and experimental methods such as laser-induced fluorescence (LIF) spectra in a free jet. ${ }^{23}$ Conclusively, benzonitrile must be characterized for the molecular structure in the bulk media, the possible molecular orientation at the liquid interface and surface energy, and the free energy of solvation with other solute moieties where it is considered as the pure or mixed solvent.

According to the studies until now, no simulation studies have been reported on benzonitrile liquid and this work is the first molecular dynamics (MD) simulation reported here. Though very recently, a study of the molecular reorientation and local structure of the benzonitrile has been done through simulations and analysis of time correlation functions. ${ }^{24}$ The purpose of the present study is to investigate the thermodynamic, structural, and transport properties of BZN liquid in the range of 293-323 K by utilizing classical MD simulation. The 
phenyl ring interaction in the stacking mode and the contribution to the liquid structure and morphology will be studied vastly. Finally, since few other simulation studies have been done to date, ${ }^{24}$ the effort is also concentrated on force field development too. To our knowledge, this research presents the first comprehensive simulation study of the thermodynamic, transport, and structural properties as well as the surface tension and molecular orientation at the liquid interface.

\section{METHOD}

To search and investigate the interaction involve and responsible for the BZN liquid structure and properties. Particularly, the stacking phenomenon through the phenyl group (being absent in counterpart acetonitrile solvent) and hydrogen bonding and their role are studied by different methods. These bring us to come up with the extensions in line with our recently conducted studies on clusters of different sizes of benzonitrile molecules that examined the effect of hydrogen bond formation on their stability. ${ }^{11}$ Also, various studies on liquid crystals and stacking, ${ }^{25-28}$ the particular effect of hydrogen bond formation $1^{29}$ are available in the literature.

2.A. Computational Details. The benzonitrile molecular structure was optimized using the Gaussian 09 program. ${ }^{30}$ Geometry optimizations of BZN monomer were performed by utilizing B3LYP functional and 6-311++G(3df,3pd) basis set. Vibrational frequencies of the neutral compound were examined to ensure that the BZN's structure has a qualified potential energy surface. Using CHELPG procedure, ${ }^{31}$ as a Gaussian 09 utility, the partial charges for BZN atoms were determined. Figure 1 display the BZN molecule and the atoms label used in all computations and simulations. The calculated partial charges are shown in Table S1 (in 
Supporting Information), and BZN geometrical parameters have been given and verified in a recent study. ${ }^{11}$

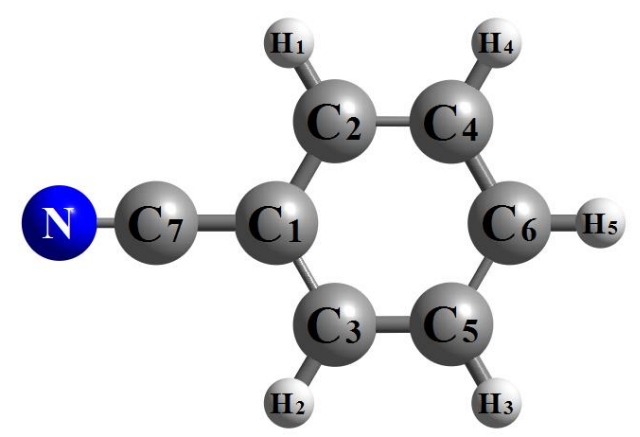

Figure 1. The structure of the optimized BZN molecule. The atom labels used throughout are shown.

All simulations were performed using the DL_POLY program version $2.17,{ }^{32}$ to investigate the structural properties of BZN liquid and calculate the bulk thermodynamic (density) and transport properties (diffusion coefficient and viscosity) at ambient pressure $\left(\mathrm{P}=1.01325 \times 10^{5} \mathrm{~Pa}\right)$. The non-polarizable optimized potentials for liquid simulation all-atom $(\text { OPLS-AA })^{33}$ force field were used in all simulations. Optimized potential includes intramolecular and intermolecular potential. The interaction potential energy function is given below:

$U_{\text {intramolecular }}=U_{\text {bond }}\left(r_{i j}\right)+U_{\text {angle }}(\theta)+U_{\text {dihed }}(\phi)$

Potential energy contribution is accounted as the followings:

$U_{\text {bond }(r i j)}=\frac{k_{r}}{2}\left(r_{i}-r_{e q}\right)^{2}$

$U_{\text {angle }}(\theta)=\frac{k_{\theta}}{2}\left(\theta_{i}-\theta_{e q}\right)^{2}$

$U_{\text {dihed }}(\phi)=\sum_{i} \frac{V_{1}^{i}}{2}\left[1+\cos \left(\varphi_{i}\right)\right]+\frac{V_{2}^{i}}{2}\left[1-\cos \left(2 \varphi_{i}\right)\right]+\frac{V_{3}^{i}}{2}\left[1+\cos \left(3 \varphi_{i}\right)\right]$

where all the terms have their usual meaning. Intermolecular interactions involve atom-atom (12-6) Lennard-Jones (LJ) potential for the van der Waals interactions and columbic electrostatic interactions between point charges centered on the atoms: 
$U_{\text {intermolecular }}=\sum_{i=1}^{N-1} \sum_{j>1}^{N}\left\{4 \varepsilon_{i j}\left[\left(\frac{\sigma_{i j}}{r_{i j}}\right)^{12}-\left(\frac{\sigma_{i j}}{r_{i j}}\right)^{6}\right]+\frac{1}{4 \varepsilon_{i j}} \frac{q_{i} q_{j}}{r_{i j}}\right.$

The potential well-depth $\varepsilon_{i j}$, and the hard-sphere diameter $\sigma_{i j}$, is determined by simple combining and mixing rule, respectively:

$\varepsilon_{i j}=\sqrt{\varepsilon_{i} \varepsilon_{j}} \quad, \quad \sigma_{i j}=\frac{1}{2} \sqrt{\sigma_{i}+\sigma_{j}}$

The partial atomic charges for this solvent were taken from our CHELPG calculations. The $\varepsilon$ and $\sigma$ parameters are given in Table S1 (in the Supporting Information). Equilibrium bond distances and angles were based on optimized geometry. The bond and angle force constants and dihedral parameters were obtained from the literature. ${ }^{34,35}$ The force field parameters are presented in Table S2 (Supporting Information).

For MD simulation, a geometry-optimized BZN structure was enfolded to make a simulation box $\left(50 \times 50 \times 50\left(\AA^{3}\right)\right)$ containing $343 \mathrm{BZN}$ molecules. The equation of motion was solved using the Verlet-Leapfrog integration algorithm. Periodic boundary conditions are applied in three dimensions. By using Ewald summation with the precision of $1 \times 10^{-5}$, Coulombic pair interaction in the form of non-bounded Lennard-Jones interaction was calculated. The potential cut-off distance of $24 \AA$ is appropriate for the simulation box involved.

The simulation was initially performed in the NVE ensemble with a short time step $(0.0001$ ps) for $1 \mathrm{~ns}$ for BZN molecules to be randomly distributed without overlapping. Then, after the heating (up to $423 \mathrm{~K}$ ) and cooling process, the simulation was continued for 5 more ns with 0.001 ps time step in NPT ensemble (for simulation of density) under Hoover thermostat and Andersen barostat algorithm. Then, the simulation was continued, to calculate transport properties, for 10 more ns in the NVT ensemble. The simulation was started from the highest temperature of the temperature range of interest (293-323 K) in $5 \mathrm{~K}$ intervals. To simulate the equilibrium and transport property, the output of one NPT ensemble was used as the input of 
the initial ensemble at the next lower temperature successively. Self-diffusion coefficient and viscosity were determined using mean square displacement (MSD) (initially calculated from the simulated trajectories of the NVT ensemble). The specification of the simulation boxes is shown in Table S3 (Supporting Information).

Also, to investigate the BZN molecular surface structure, $540 \mathrm{BZN}$ molecules were simulated in a cubic box of the sizes $50 \times 50 \times 50\left(\AA^{3}\right)$. After equilibration, the ensemble was extended in the $z$-direction to $150 \AA$ to create a liquid slab with a final thickness of about $50 \AA$. Similarly, MD simulation was performed in the NVE ensemble for $1 \mathrm{~ns}$ before performing the heating-cooling cycle. After cooling the ensemble down to 298K, MD simulation was continued in the NVT ensemble for 20 more ns.

\section{RESULTS AND DISCUSSION}

The liquid bulk properties including structure, thermodynamics, and transport as well as the surface properties have a fundamental relationship with the molecular structure. We present the result of computational studies that incorporate the stacking phenomena of the BZN phenyl ring.

\section{A. Bulk liquid benzonitrile}

3.A.1. Density and Force Field Assessment. The liquid density (equivalently the hardsphere diameter) and intermolecular forces are the most relevant quantity in the thermodynamic of a fluid from which the basic parameters of the equation of state can be derived. Fortunately, liquid density can be measured accurately by experimental methods easily. Since the liquid density depends on the intermolecular forces too, this single parameter is usually utilized to test the validity of the force field applied in the simulation. Table 1 contains the simulated density of liquid benzonitrile in the range of 293 to $323 \mathrm{~K}$ and 1 atm pressure. The densities 
obtained are in good agreement (with a maximum deviation of $0.29 \%$ ) compared to the experimental densities ${ }^{36,37}$ over the whole temperature. The result of the simulation can be tested visually and verified numerically with regards to literature experimental data (See Table 1 and Figure 2). We will discuss the small deviation from the linear behavior of the temperature dependence of density centered at $308 \mathrm{~K}$.

Table 1. Simulated densities (at one atmosphere) and comparison with experimental values. ${ }^{36,37}$

\begin{tabular}{|c|c|c|c|}
\hline \multirow{2}{*}{$T(\mathrm{~K})$} & \multicolumn{3}{|c|}{$\rho,(\% \mathrm{Dev} .)^{*}\left(\mathrm{gr} / \mathrm{cm}^{3}\right)$} \\
\cline { 2 - 4 } & \multirow{2}{*}{ Simulation } & {$[36]$} & Experimental \\
\cline { 2 - 4 } & & - & - \\
\hline 293.15 & 1.0028 & $1.0009(0.27)$ & $1.0006(0.24)$ \\
\hline 298.15 & 0.9982 & $0.9964(0.21)$ & $0.9963(0.20)$ \\
\hline 303.15 & 0.9943 & $0.9919(0.29)$ & $0.9918(0.28)$ \\
\hline 308.15 & 0.9890 & $0.9872(0.09)$ & $0.9862(0.01)$ \\
\hline 313.15 & 0.9863 & - & $0.9830(0.01)$ \\
\hline 318.15 & 0.9829 & - & - \\
\hline 323.15 & 0.9790 & \multicolumn{3}{|l}{} \\
\hline$* \%$ Dev $=100 \times\left(\left|\rho_{\text {exp }}-\rho_{\text {sim }}\right|\right) / \rho_{\text {exp }}$ & \multicolumn{5}{|c}{} \\
\hline
\end{tabular}

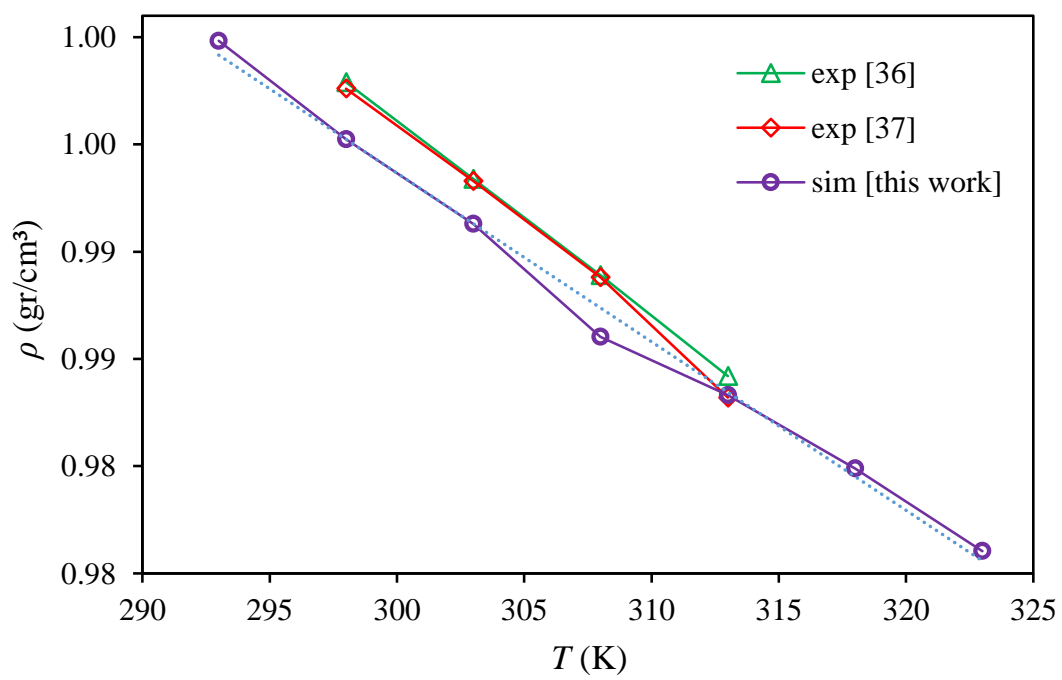

Figure 2. Comparison of the temperature-dependent density of the benzonitrile liquid. The dotted line is the linear fit. 
3.A.2. Liquid Structure. The investigation for the structural properties of benzonitrile liquid involves the simulated atom-atom radial distribution function (RDF). This is under the condition that, particular hydrogen bonding structures confirmed based on clusters formation of different sizes have been already established ${ }^{11}$ as shown in Figure 3 . We study the $g(r)$ 's through $\mathrm{CN}$ group (employing $\mathrm{N}$ atom) and different $\mathrm{H}$ atom sites at equilibrium as shown in Figure 4. The first peak indicates that the correlation of the $\mathrm{N}$ atom with different H's (ortho, meta, and para) are strong and of low dynamics at short ranges. The first peaks of $\mathrm{N} \cdots \mathrm{H}_{1}\left(\mathrm{H}_{2}\right)$ interacting at $~ 2.945 \AA$ Andicate (both equally) strong correlations and lowest dynamics at short range (compared to other cases in Figure 4) and persist well in the long-range (second peak). The fact that a conformer comprising, for instance, one benzonitrile dimer forming simultaneously two strong $\mathrm{H}$-bonds (like $-\mathrm{C} \equiv \mathrm{N} \cdots \mathrm{H}($ ortho $)$ ) can thoughtfully account for this.

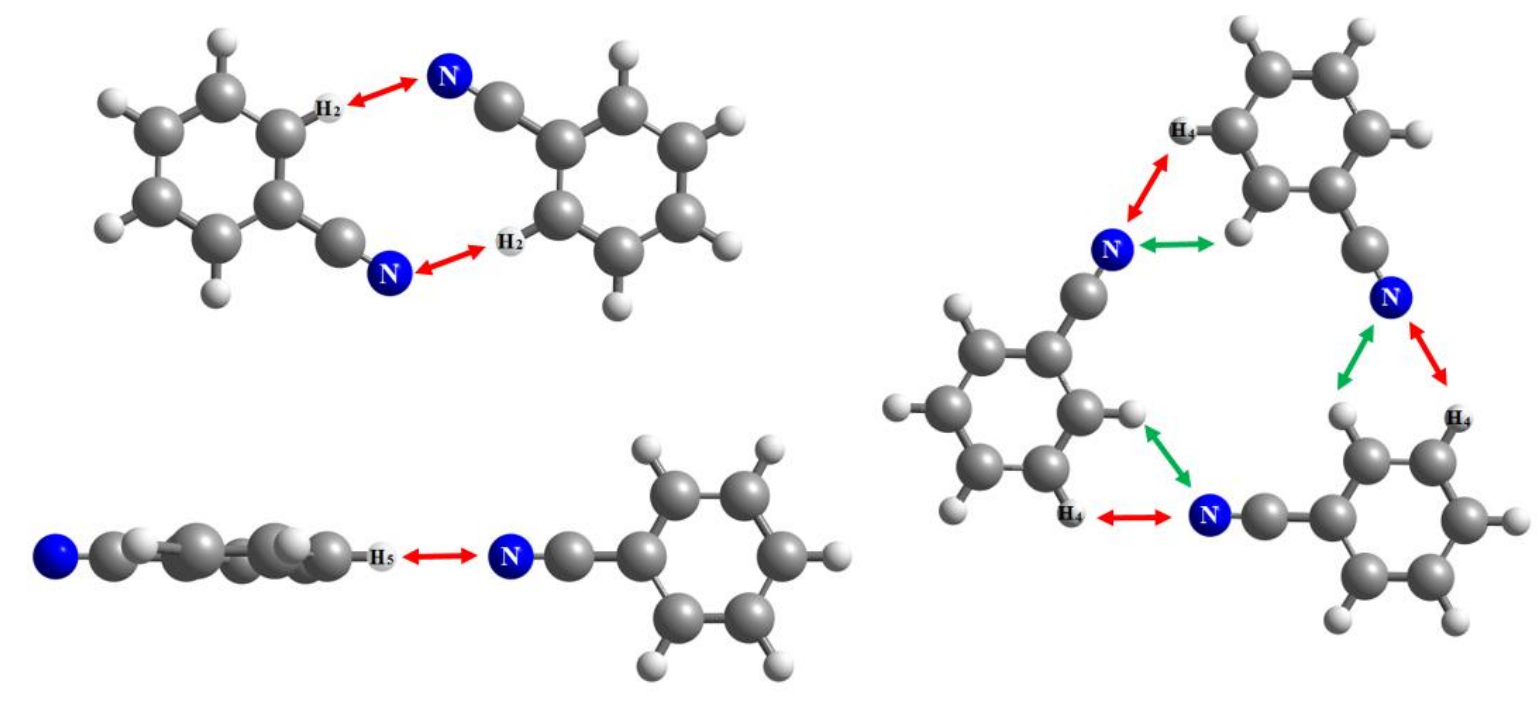

Figure 3. The distinguished hydrogen bonding in the dimmer and trimer BZN clusters.

The first peak is generally well-shaped in a similar way for the three different $\mathrm{H}$ atoms while peak heights are slightly different in the order of meta- $\mathrm{H} \approx>$ para $-\mathrm{H} \approx>$ ortho $-\mathrm{H}$ and the peak width is in the order meta- $\mathrm{H} \sim>$ para $-\mathrm{H}>>$ ortho-H. These peaks located in the 2.8 - 
$2.9 \AA$ domain could be attributed to the short-range electrostatic interactions enhanced due to hydrogen bonding's of moderate strength, being in good agreement with recent $a b$ initio studies done on benzonitrile by considering benzonitrile clusters up to tetramers. ${ }^{11}$

Owing to such behavior, we also investigated the temperature dependence of $g(r)$ 's in the range of 293-323 K and focused on peak heights as plotted in Figure 5. As can be seen, the peak height is not considerably different among different cases of $-\mathrm{C} \equiv \mathrm{N} \cdots \mathrm{H}$ involvement (being approximately 0.04 ). Therefore, the lower dynamics of $-\mathrm{C} \equiv \mathrm{N} \cdots \mathrm{H}_{1}\left(\mathrm{H}_{2}\right)$ brought up a conclusive assessment of the possible H-bonding's strength and its influential value on liquid structure in the order of ortho-H >> meta-H $\sim>$ para-H. The subsequent peaks (particularly, the second peaks) are an indication of rather good coordination of dipoles at long ranges, whereas $-\mathrm{C} \equiv \mathrm{N} \cdots \mathrm{H}_{1}\left(\mathrm{H}_{2}\right)$ shows a second peak appreciably stronger than others. For more investigations, other analyses like dimer existence autocorrelation functions (DACF) and the evaluated structural condition were calculated by the TRAVIS package. ${ }^{38}$

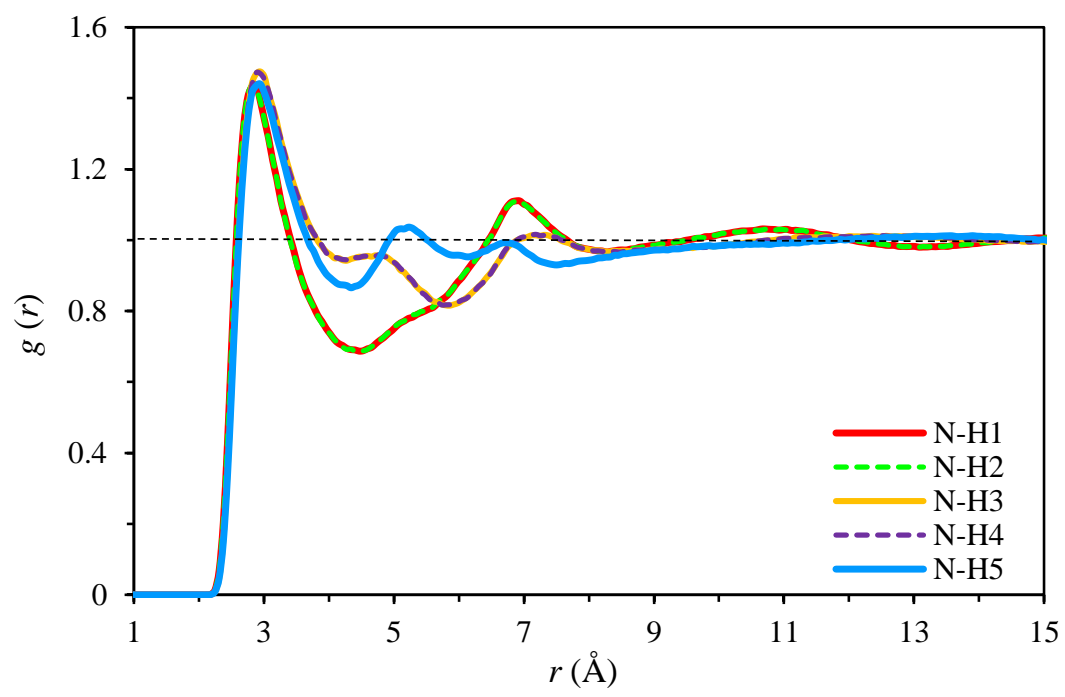

Figure 4. The simulated radial distribution function of $\mathrm{N}$ and different $\mathrm{H}$ atomic sites for benzonitrile liquid at $298 \mathrm{~K}$. 


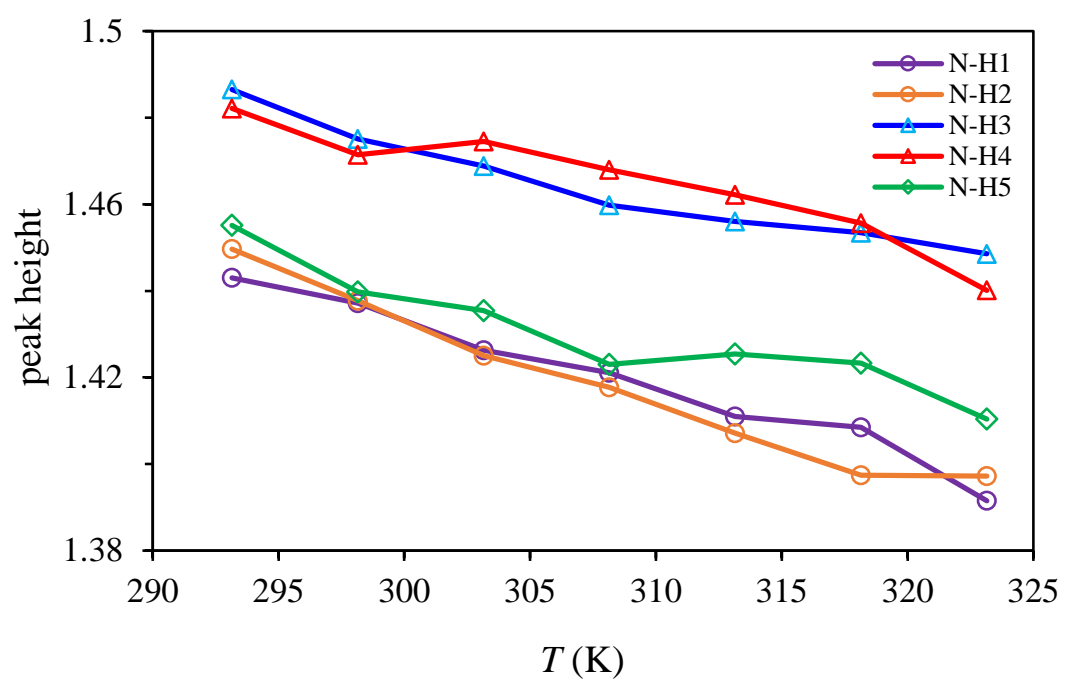

Figure 5. Temperature dependence of the peak heights of the radial distribution functions.

In the DACF, we can calculate the lifetime that two specific atoms spend next to each other by integrating the dimer existence autocorrelation functions at $298 \mathrm{~K}$ and the results are given in Table 2. The results show that the lifetime of the interaction between the ortho- $\mathrm{H} \cdots \mathrm{N}$ is well higher than others. Also, the percentage of the simulation time that two atoms experience a strong correlation (Evaluated structural condition) has been calculated in all temperatures. By examining Figure 6, an important conclusion brought about by the MD simulation of bulk BZN is that the lifetime of the $\mathrm{N}$ atom interacting with the $\mathrm{H}$ atom is in the order of ortho- $\mathrm{H}>$ meta$\mathrm{H}>$ para $-\mathrm{H}$.

Table 2. The calculated average lifetime between $\mathrm{N}$ and different $\mathrm{H}$-atoms.

\begin{tabular}{|l|c|}
\hline \multicolumn{1}{|c|}{ atoms } & Lifetime $(\mathrm{ps})$ \\
\hline ortho $-\mathrm{H} \cdots \mathrm{N}$ & 9.9 \\
\hline meta $-\mathrm{H} \cdots \mathrm{N}$ & 3.8 \\
\hline para $-\mathrm{H} \cdots \mathrm{N}$ & 1.2 \\
\hline
\end{tabular}




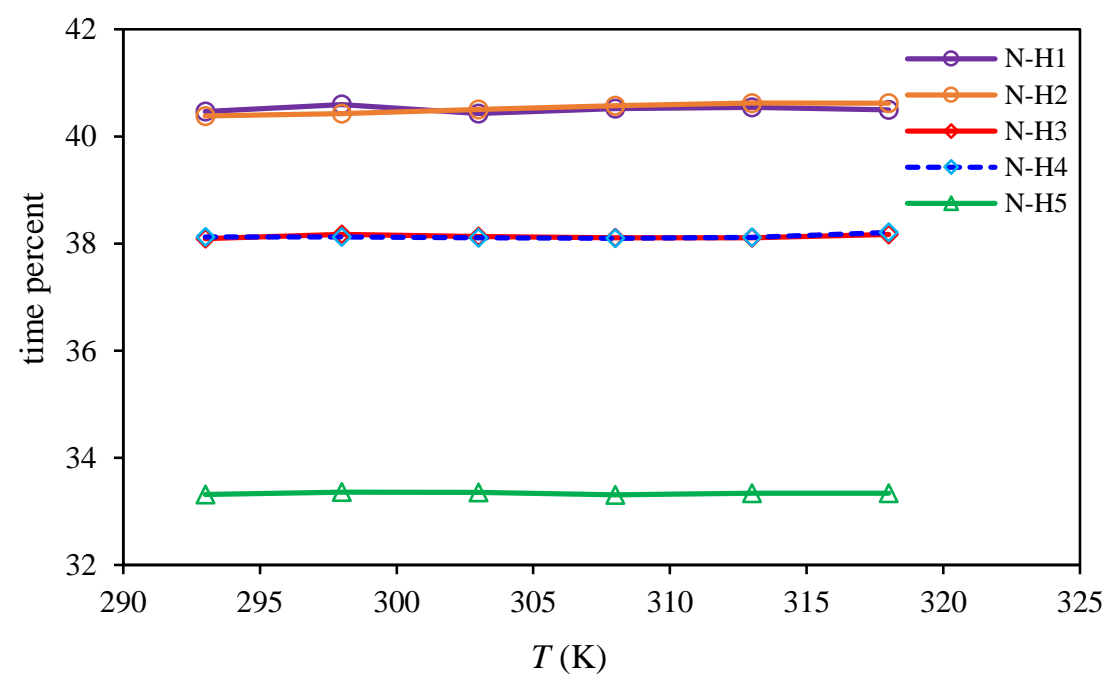

Figure 6. Simulated temperature dependence of structural interrelation for benzonitrile liquid.

Considering the results in Figures 4 and 5, and the analyzes shown in Table 2 and Figure 6, the strength of $-\mathrm{C} \equiv \mathrm{N} \cdots \mathrm{H}$ correlation is in order of ortho $-\mathrm{H}>$ meta $-\mathrm{H}>$ para $-\mathrm{H}$ at all temperatures. These results are in good agreement with reported simulation studies. ${ }^{24}$ Therefore, it can be concluded that the most interaction between benzonitrile molecules occurs through ortho-H $\cdots \mathrm{N}$ with low dynamic while lasting longer. Earlier ab initio calculations have shown that the H-bonding in BZN through ortho- $\mathrm{H} \cdots \mathrm{N}$ is well-contributed specifically by the both $\sigma$ - and $\pi$-bonding. ${ }^{11}$ Therefore, the possible H-bonding types demonstrated for different clusters, ${ }^{11}$ for example in Figure 3, confirm the above trend in the correlation strength.

\section{A.3. Structural Properties: Combined Distribution Function. Further structural} analysis was elaborated by utilizing the combined distribution function. Such a goal was achieved by mapping combined radial and angular distribution functions using the TRAVIS package. ${ }^{38}$ Therefore, a counter of angular distribution functions (ADF) versus RDF made it possible to map and understand by what angle $(\theta)$ does $-\mathrm{CN}$ group approach $(r)$ the $\mathrm{H}$ atom (Figure 7(a)) while is keeping an appreciable correlation. The results illustrated in Figure 7(b) is representing a well-defined and precise ortho- $\mathrm{H} \cdots \mathrm{N}$ correlation, with a maximum between 
$\theta=120^{\circ}$ and $135^{\circ}$. For the meta and para cases, the characteristic simulated angles $\theta \sim 180^{\circ}$

(Figures $7(\mathrm{c})$ and (d)) are revealing and confirming the earlier finding ${ }^{11}$ that the para- $\mathrm{H}$ and the meta-H interact with $\mathrm{N}$ atom through $\sigma$-bond and $\pi$-bond formation, respectively.
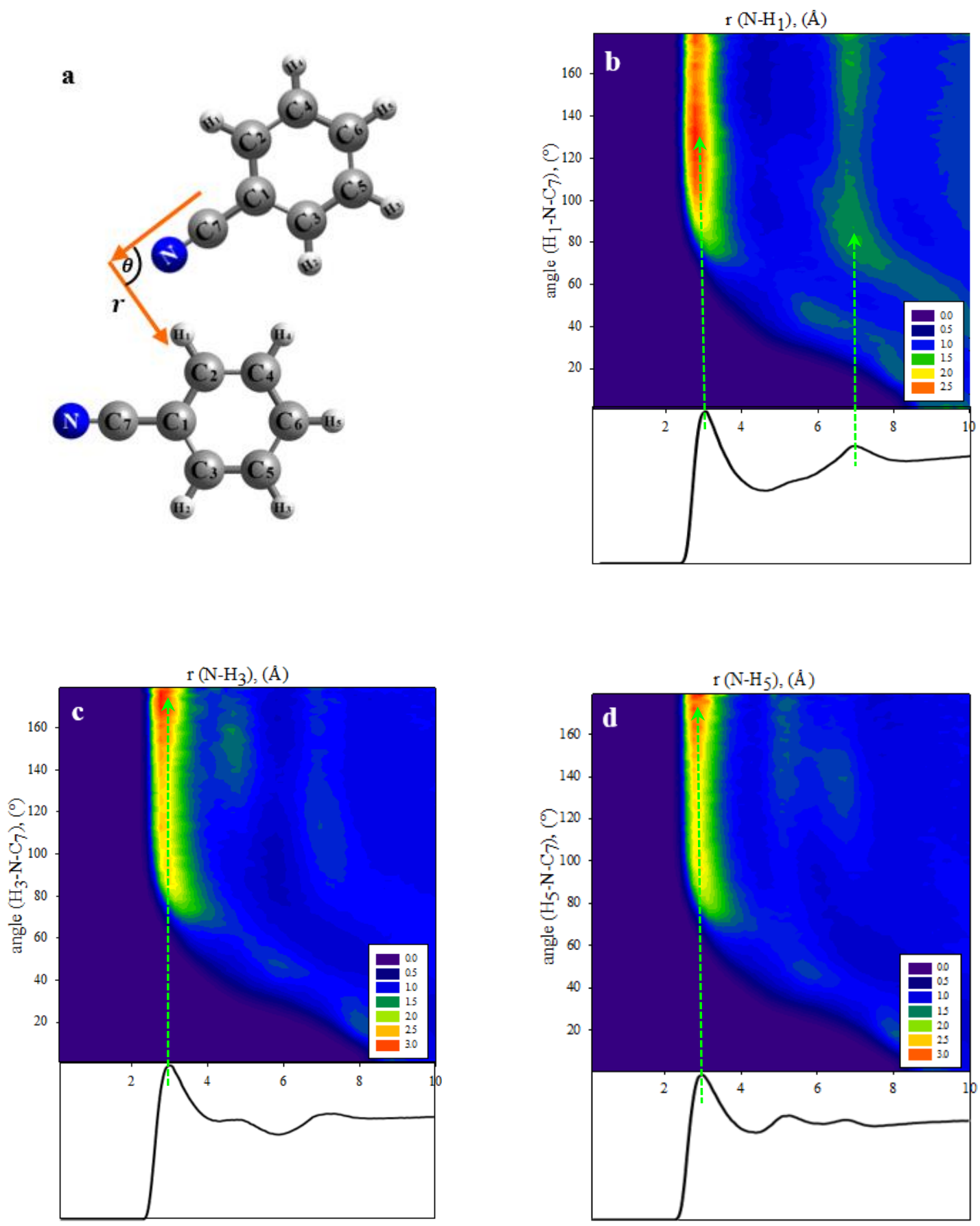

Figure 7. (a) Representation of angle $\theta$ and distance $r$, and (b)-(d) the combined distribution function for hydrogen bonding between $\mathrm{N}$ and ortho-, meta-, para-H atoms. 
3.A.4. Transport Properties. In addition to calculating the equilibrium thermodynamic properties (leading to force field development) and the liquid structural feature, the dynamics of the BZN molecules in the liquid state can be investigated by the simulation of transport properties. Therefore, the mean square displacement (MSD) and the self-diffusion coefficient of benzonitrile liquid were calculated and shown in Figures 8 and, 9, respectively. The MSD was calculated from the knowledge of coordinates of the particles during the simulation in the NVT ensemble:

$M S D=\frac{1}{N}\left\langle\sum_{i=1}^{N}\left|r_{i}(t)-r_{i}(0)\right|^{2}\right\rangle$

where $r_{i}(t)$ is the center-of-mass location of the particle $i$ at time $t$, and $N$ is the number of particles.

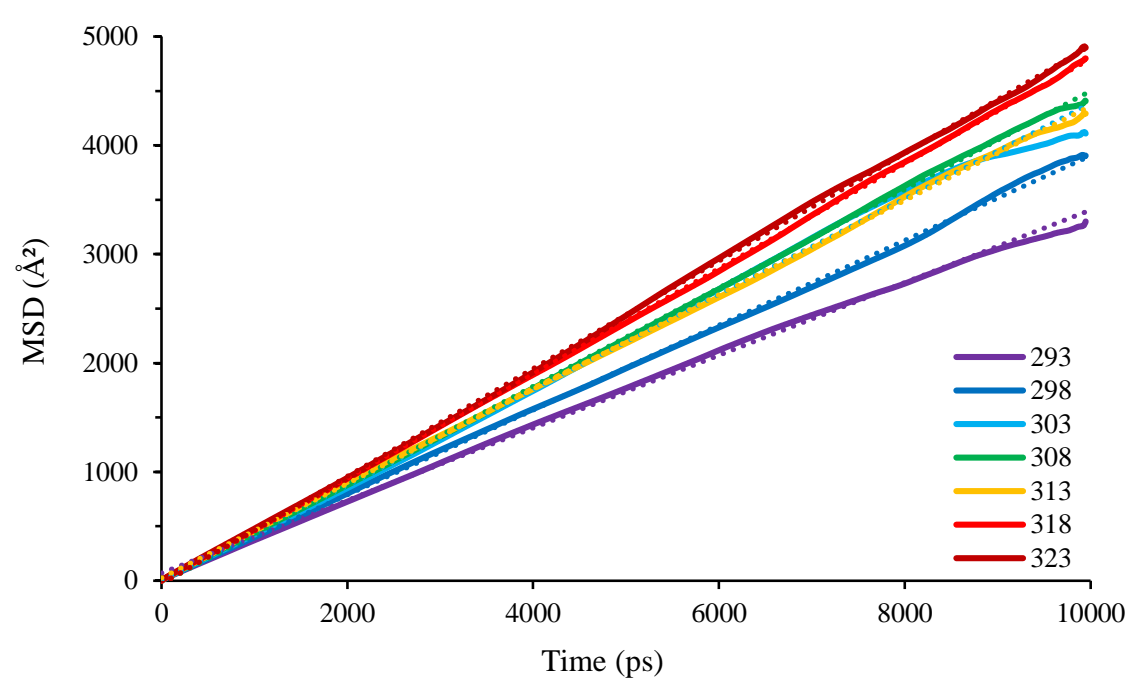

Figure 8. Simulated MSD of the center of mass of benzonitrile liquid at different temperatures. The thick lines are composed of a copious number of overlapping data points. Dotted lines are the linear fits.

Self-diffusion coefficient $\left(D_{i}\right)$ represents the microscopic liquid dynamics and were obtained from the linear regime of $M S D$ curves at long simulation time using the Einstein relation:

$D_{i}=\frac{1}{6} \lim _{t \rightarrow \infty} \frac{d}{d t}\left\langle\left|r_{i}(t)-r_{i}(0)\right|^{2}\right\rangle$ 
Self-diffusion coefficients for the benzonitrile liquid were calculated (from the simulation for $10 \mathrm{~ns}$ ) in the temperature range of 293 to $323 \mathrm{~K}$ are shown in Figures 9.

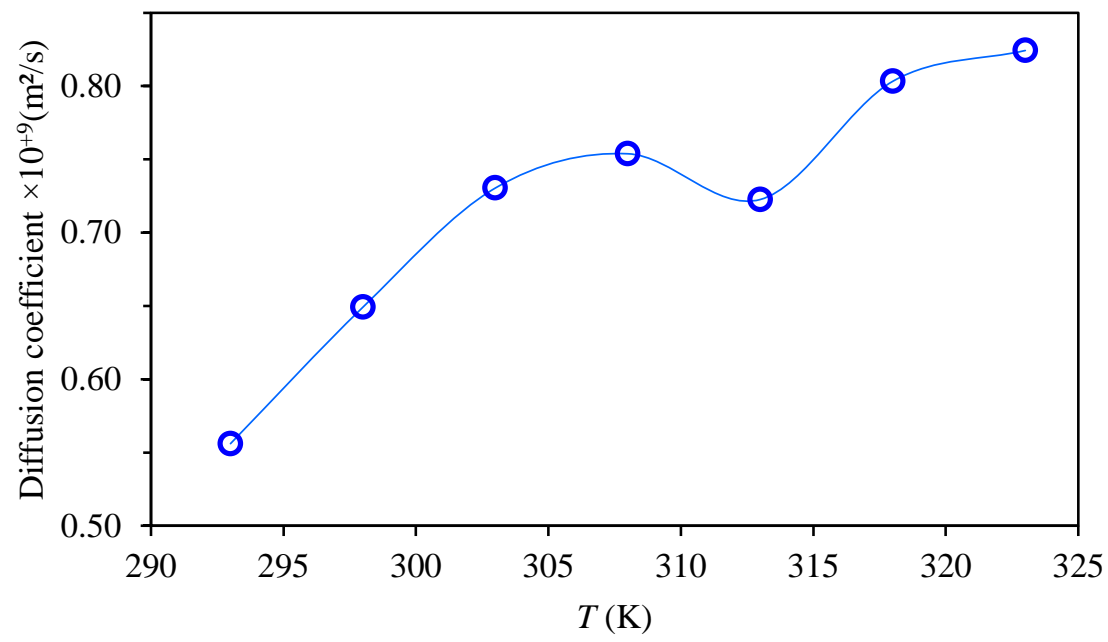

Figure 9. The temperature dependence of the calculated self-diffusion coefficient of benzonitrile liquid.

As seen in Figure 9, self-diffusion for BZN liquid increases (almost quadratically) with increasing temperature while showing a fluctuation at $313 \mathrm{~K}$. In addition, we calculated the viscosity of benzonitrile liquid (Figure 10) to test our refined force field parameters. For characterization and investigation of the microscopic dynamics of the liquid bulk, the viscosities of BZN were estimated using the Stokes-Einstein equation,

$\eta=\frac{k_{B} T}{c \pi \alpha D_{i}}$

where $k_{B} T$ is the thermal energy at temperature $T, \alpha$ are the molecular diameter and $c$ is a parameter characteristic of the liquid. The value of $c$ depends on the molecular type and is often adjusted (usually between 4 and 6 ) for the best result. For benzonitrile liquid in this work, $c=$ 6 was adopted. To determine a mean value $\alpha$, we employed quantum mechanics using the Gaussian 09 program $^{30}$ and calculated the volume of the benzonitrile molecule. The simulated viscosities are plotted in Figure 10 and compared with the experimental data. Like all liquids, the simulated BZN viscosity decreases monotonically as the temperature increases, though, 
consistent with the profile of the diffusion coefficient (Figure 9), fluctuates at about $313 \mathrm{~K}$. Quite interestingly, a fluctuation is seen in the experimental viscosity of benzonitrile, ${ }^{23}$ in the same temperature range as shown together with the simulations in Figure 10. Therefore, the fluctuation is part of the viscosity profile, whereas confirming the validity and accuracy of the simulation of viscosity in detail. Under the condition that no interpretation has been given to the observed experimental viscosity to date, we got the impression that the nature of these fluctuations root in the factors that can be clarified by the simulation results. The conjecture is that the fluctuation in viscosity can be attributed to the specific conformation occurring at certain temperatures that leads to the stacking of benzene rings of the benzonitrile molecules as will be discussed in detail in the next section.

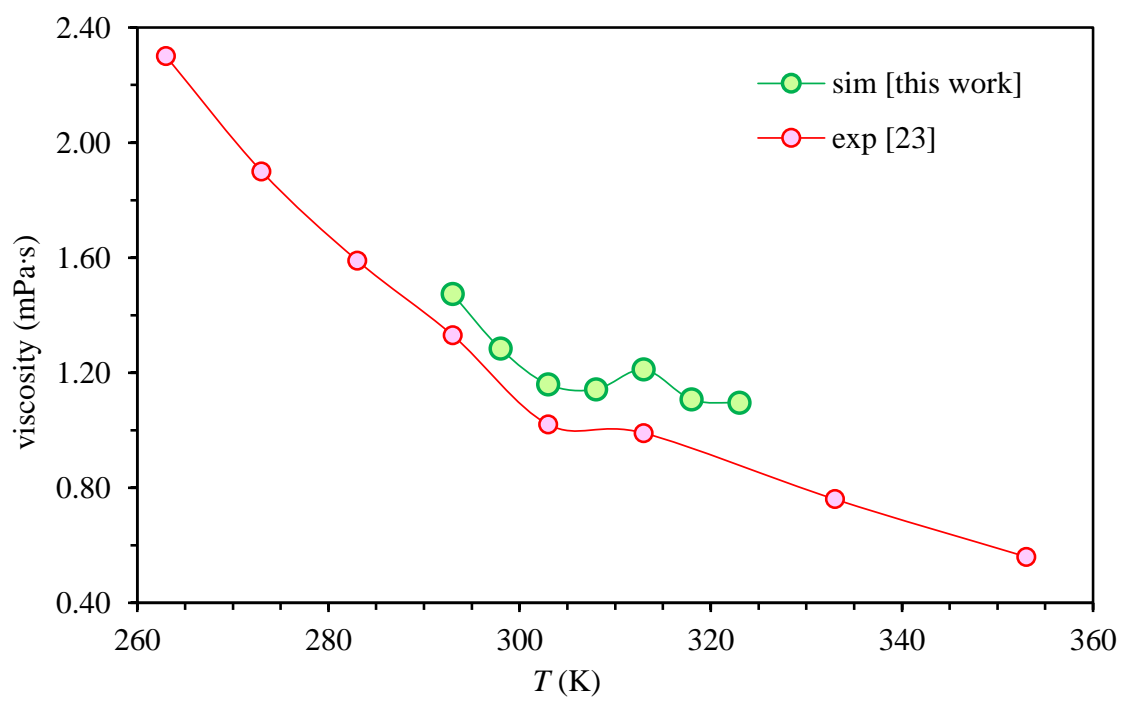

Figure 10. The calculated viscosity of benzonitrile liquid at different $T$. Lines are trend lines.

3.A.5. Molecular Stacking in Benzonitrile. To date, several studies have been performed on $\pi-\pi$ interactions in liquid benzene and some of its derivatives, such as benzonitrile. ${ }^{39} \mathrm{In}$ these compounds, due to the dispersion attraction between the positively charged rings and the $\pi$ electrons, the two rings may combine in a face-to-face sandwich configuration. Certainly, factors such as electron-withdrawing and electron-donating of the benzene ring having substituted with functional groups would affect this behavior. For example, in benzonitrile, the 
electron-withdrawing substituent $-\mathrm{C} \equiv \mathrm{N}$ group helps to enhance the configuration with $\pi$ stacking interaction as a result of electron withdrawn from the conjugated $\pi$ bonds and thus reducing the electrostatic repulsion. For this reason, a 100 ps simulation was performed (Hyperchem 7) $)^{40}$ at ambient temperature on benzonitrile clusters of various sizes. As shown in Figure 11, the final structures led to clusters, all interacting in a ring stacking mode.

a

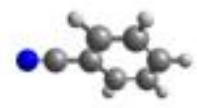

b

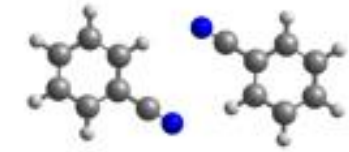

c

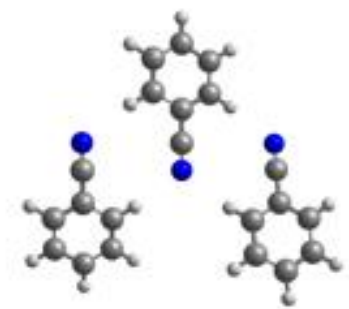

d
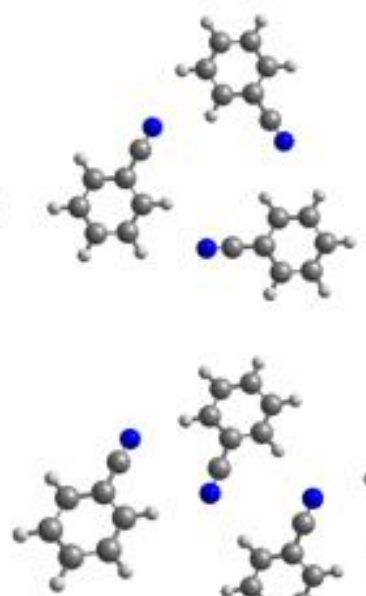
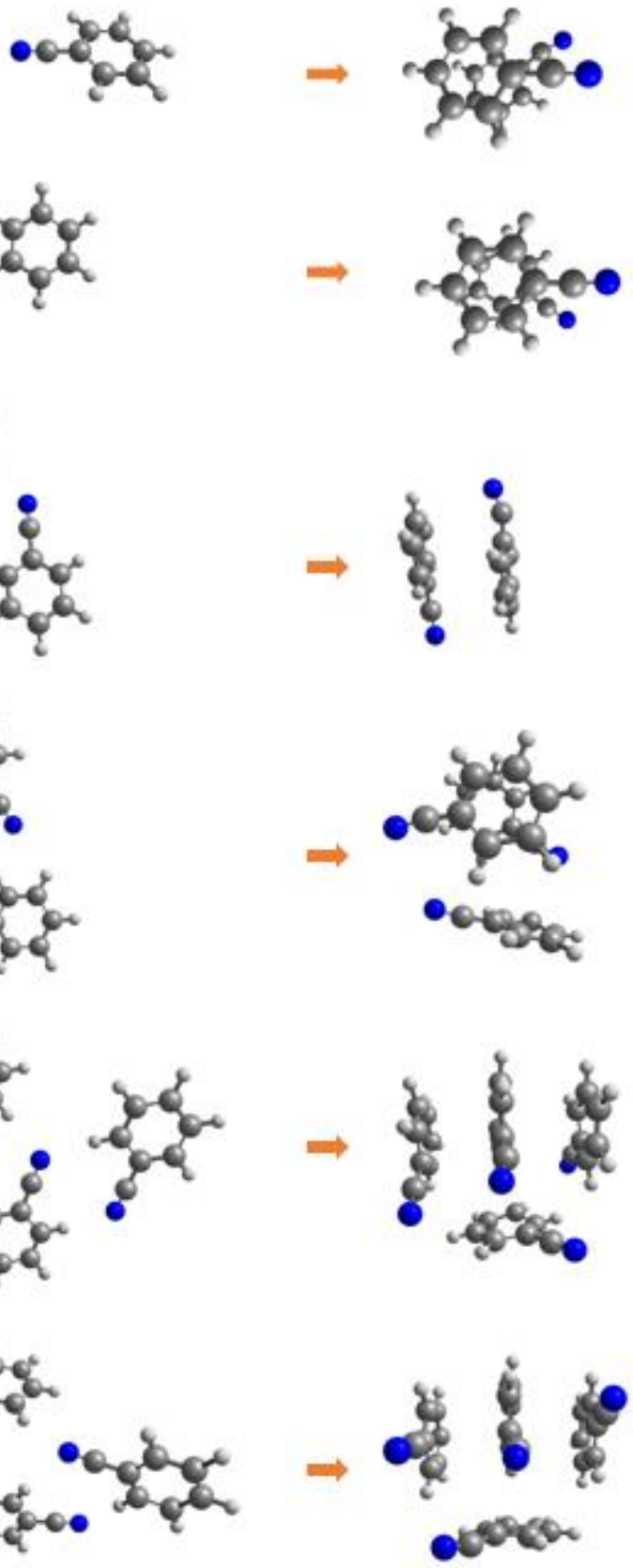

Figure 11. The appearance of stacking in clusters of different sizes of benzonitrile with initial configuration, (a) dimer-1, (b) dimer-2, (c) trimer-1, (d) trimer-2, (e) tetramer-1 and (f) tetramer-2. 
For quantifying the stacking behavior, the radial distribution functions between the center of masses of BZN molecules in liquid bulk were analyzed by utilizing a $10 \mathrm{~ns}$ simulation at different temperatures. Although the stacking assessment may be done by using radial distribution function between different parts of the molecules while are correlated in stacking mode, we found the assessment based on the center of mass produces results for best comprehending in our presentations. This choice indeed puts all things together for visual inspection of the basic facts, relevantly and correctly. In another sense, this produces a module for angle-dependent pair correlation function in a relevant way. (See Figure 12 for the position of the center of mass, near the $\mathrm{C} 1$ atom).

This leads to a rather well-defined sharp peak (shown in Figure 13 for different temperatures) that implies a good correlation between the centers of mass of the BZN molecules. Indeed, we may not be encountered with a straightforward analysis of the existing intrinsic complex stacking because of the extensive overlap of the correlation functions arising from dimer, trimer, and possible tetramer stacking conformers. From the first main peak occurring at $6.23 \AA$ with appreciable dynamics, it can be inferred that distance $r(=6.23 \AA)$ matches the correlation of the first benzonitrile molecule with the third one while three molecules are stacked one on top of the other one.

A shoulder inappropriately masked underneath the main peak contains attractive information due to its marked distinguishing position being at about 3.5-4.0 $\mathrm{\AA}$. The distance of the closest approach of the masked peak (at about $3.38 \AA$, see Figure 13) is notably close to the spacing between two graphene sheets. ${ }^{41}$ Beyond the first main peak, a second peak (hardly seen due to extensive overlapping) at a long distance can be attributed to the correlation with the fourth BZN molecule in a tetramer positioned equidistance at about $9.90 \AA$ (in stacking mode). All these trends are challenging, though, undertakable naively by noting that the overall pair correlation curve (Figure 13) is the summation (overlapping) of several pair correlation curves 
each containing correlation information of stacking of BZN cluster of different sizes and structures sorts. These outcomes are quantitatively supporting the impression that each ring plane tends to interact likely in a parallel stacking mode and give a hint to the importance of the angle dependence of the correlations.

So, an analysis based on the combined orientational distribution function was performed on the bulk liquid BZN simulated at different temperatures. Accordingly, a hypothetical vector from the center of mass to the center of the benzene ring laying in the BZN molecule plane (Figure 12) was defined, and its angular distribution among different molecules in the ensemble was calculated to estimate the extent and role of the relative molecular orientation of the clusters. Combining this vector orientational distribution with the radial distribution function produces maps suitable for deconvoluting the complex radial distribution function of Figure 13, which would be otherwise very difficult (if not impossible) by the conventional deconvolution mapping popular for the analytical curves

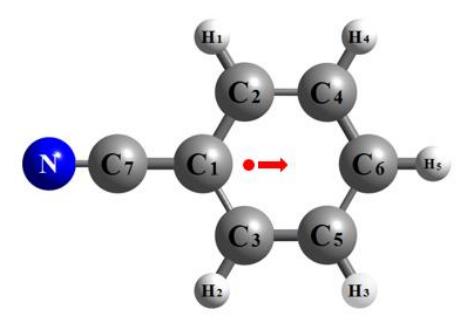

Figure 12. The position of the center of mass and the hypothetical vector from the center of mass to the ring center in the BZN molecule. 


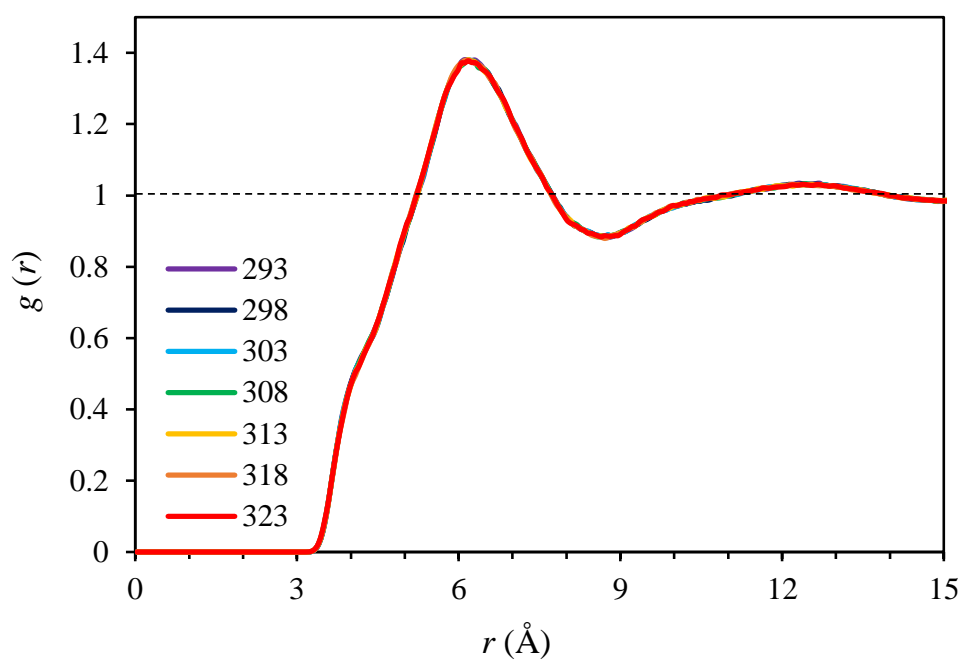

Figure 13. The radial distribution functions between the center of masses of BZN molecules in the liquid bulk at different temperatures. The $g(r)$ 's values are extensively overlaid at all different temperatures considered.

The combined distribution function (at $298 \mathrm{~K}$ ) shown in Figure 14 indicates that the correlation between the center of mass of two adjacent rings in stacking mode (in a dimer, trimer, ...) is strongest where the most probable angle between their in-plane vectors (shown in Figure 12) is centered strictly at $180^{\circ}$ and $0^{\circ}$. However, the two vectors oriented with $180^{\circ}$ are much more probable than the $0^{\circ}$. These quantitative findings are in good agreement with the quantum mechanical studies ${ }^{42}$ as are shown schematically in Figure 15 . Furthermore, the results distance-wise are in good agreement with the 3.2-3.4 $\AA$ distance reported by quantum mechanical calculation. ${ }^{42}$

According to Figures 14 and 15, the state that the planes of the BZN molecule stack together are characterized here by the angle between the in-plane-vectors, having preferentially accessed to in the order of $180^{\circ}>0^{\circ}>>90^{\circ}$ (Figure $15(\mathrm{a})$ ). In short, based on the deconvolution scheme followed, the two adjacent BZN molecules be stacked and correlated effectively to make a stable dimer if they have achieved an anti-parallel in-plane-vector orientational conformation, in good agreement with the recent simulation reported. ${ }^{24}$ 


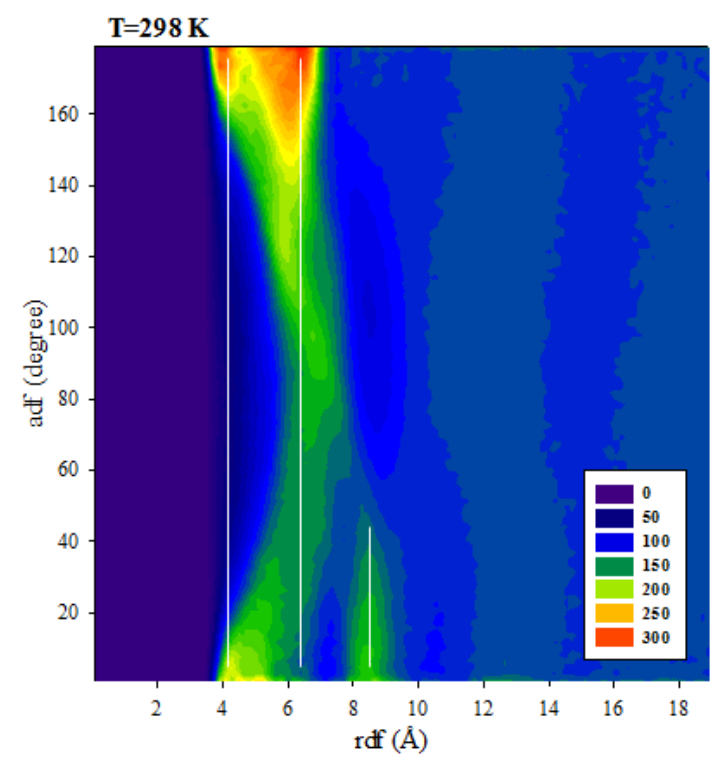

Figure 14. The combined angle distribution function for the in-plane-vector versus distance corresponding to RDF (shown in Figure 13) at $298 \mathrm{~K}$.

It is worth noting, due to the effective repulsion interaction of the cyano group at short ranges, the benzonitrile molecules in the predicted most probable orientation of $180^{\circ}$ do not overlap exactly and show slight shifting as shown in (Figure 15(a)). By calculating the radial distribution functions for $-\mathrm{C} \equiv \mathrm{N} \cdots \mathrm{C}_{(1-7)}$ in anti-parallel in-plane-vector orientation (Figure 16), it is found that the interaction of $\mathrm{C} 4$ and $\mathrm{C} 5$ atom with $\mathrm{N}$ atom with the characteristics peak maximum at $3.8 \AA$ and the distance of the closest approach of $\sim 3.0 \AA$.

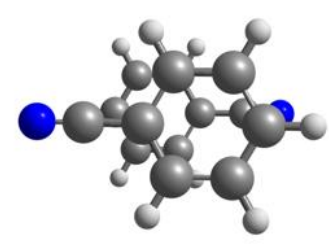

a

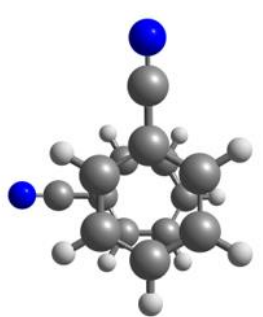

b

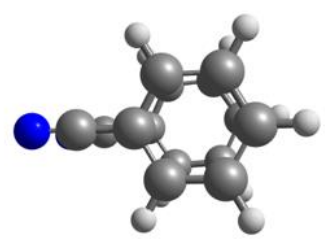

c

Figure 15. Schematic representation of the most probable states for BZN molecules in stacking mode in-plane orientation angle of (a) $180^{\circ}$, (b) $90^{\circ}$, and (3) $0^{\circ}$. 


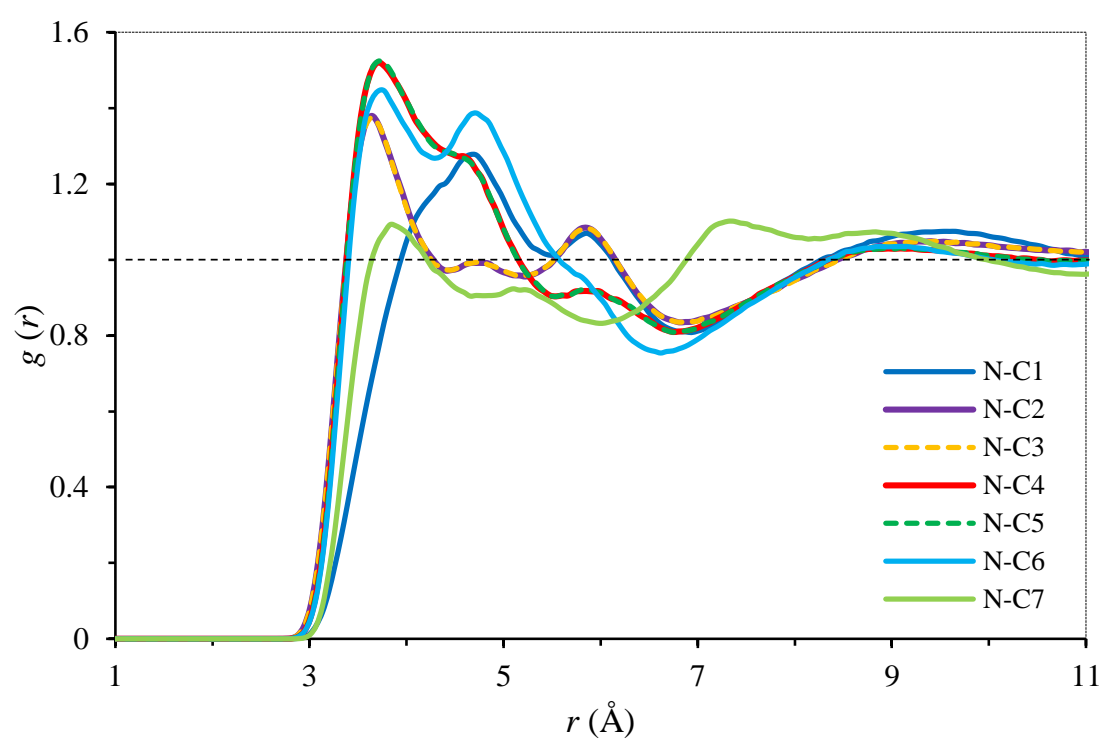

Figure 16. The simulated radial distribution function of $-\mathrm{C} \equiv \mathrm{N} \cdots \mathrm{C}_{(1-7)}$ for BZN liquid at $298 \mathrm{~K}$.

Further detailed analysis on the simulation performed at different temperatures up to $323 \mathrm{~K}$ is shown in Figure 17, according to which, the stacking occurs at all temperatures. Here, (the color coding in) Figure 17 indicates the $\pi-\pi$ stacking interaction of BZN rings occurs more effectively by turning into an anti-parallel configuration with high probability (within a limited distance and low dynamics) at the characteristic temperature $T=308 \mathrm{~K}$.
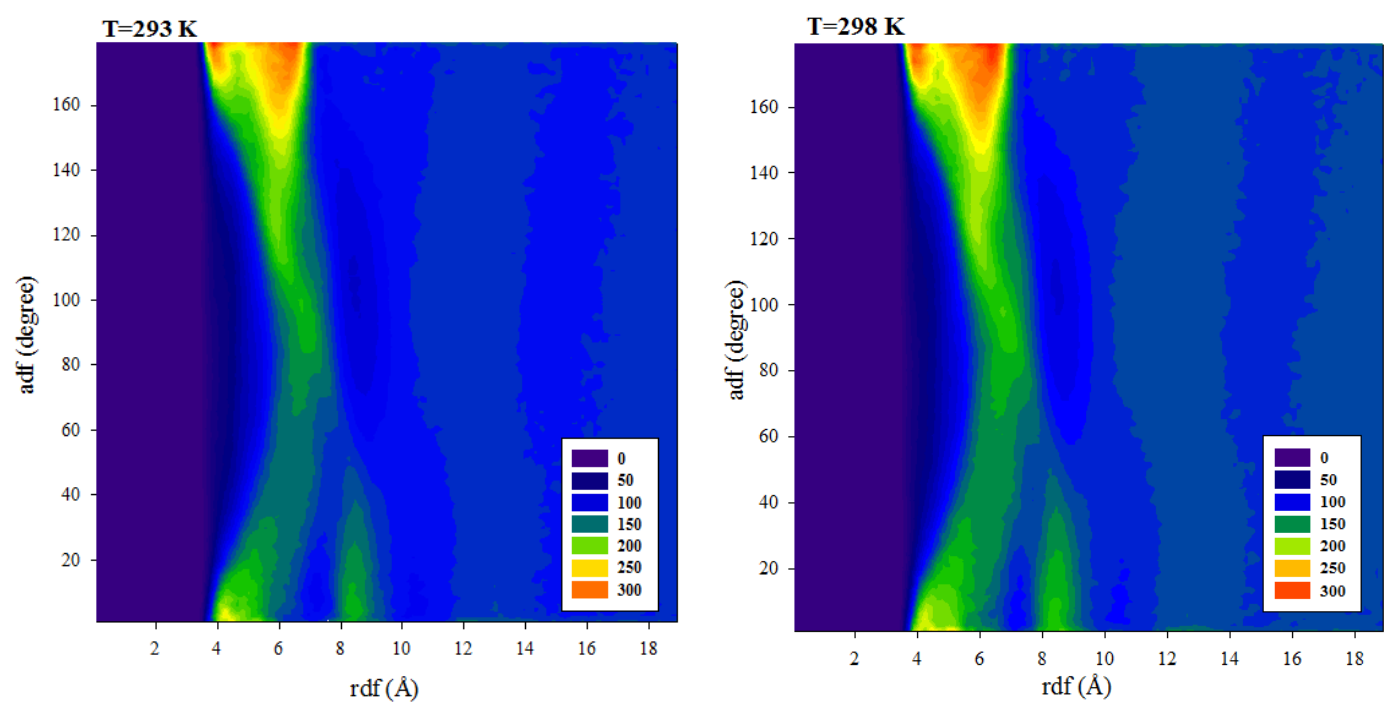

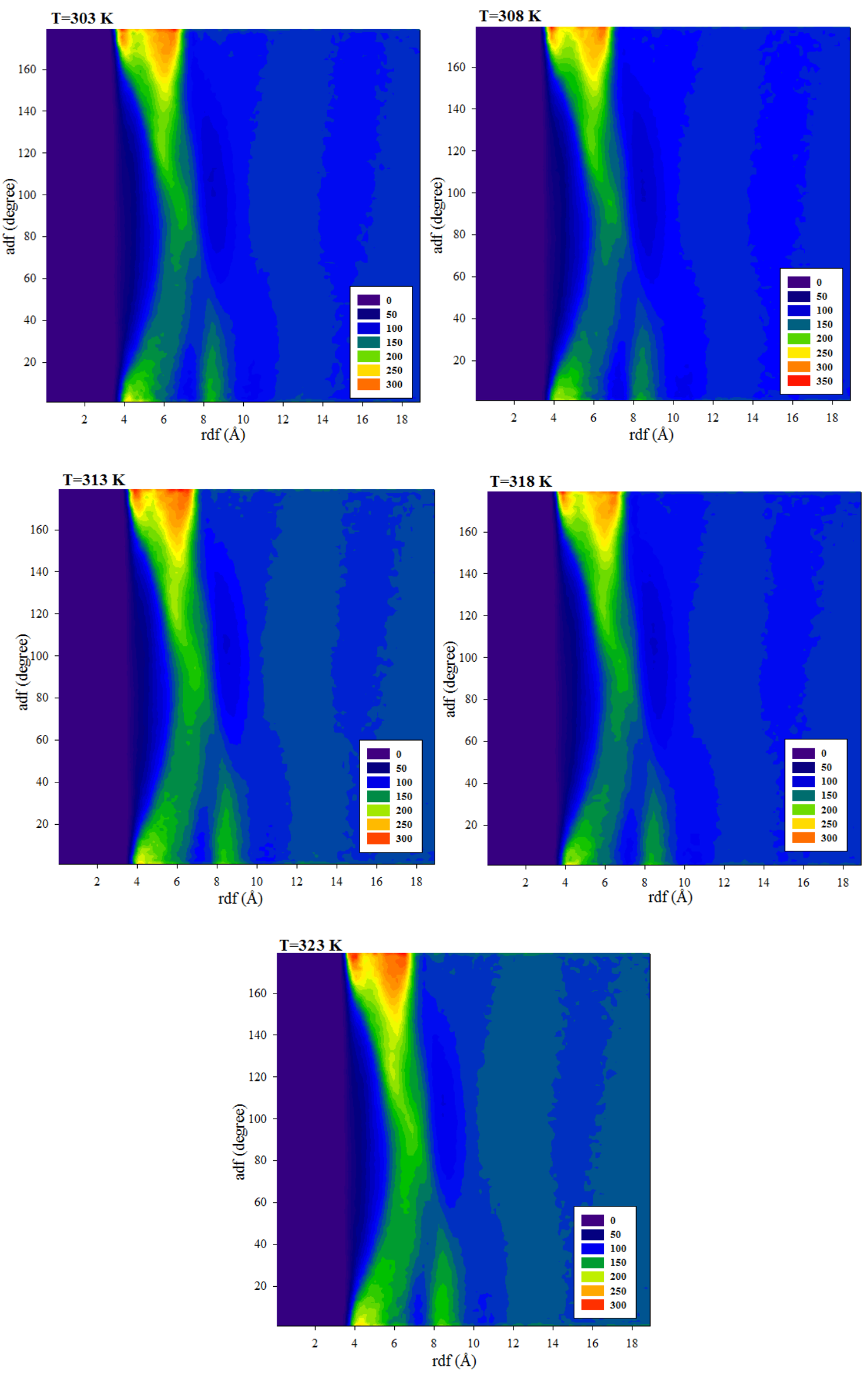

Figure 17. The same as Figure 14 but for different temperatures (293-323 K). 
To unravel the dependence of BZN stacking on temperature, a refined orientational analysis was done by selecting two hypothetical plates on the BZN molecule (Figure 18), one involving the ring only and one involving the ring and the $\mathrm{CN}$ group, and the angle between the vectors perpendicular to these plates at different temperatures was calculated. For this purpose, the angles formed between two vectors defined by vector $\mathrm{V} 1$ in reference to vector $\mathrm{V} 2$ are calculated by Travis software ${ }^{38}$ and by analyzing the radial distribution function. For the angular distribution function (ADF) analysis at each temperature, the occurrence for the most probable angle (of V1-V2) was calculated and shown in Figure 19.

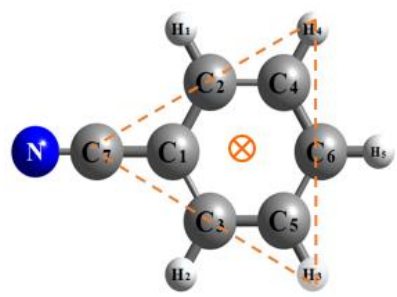

V1

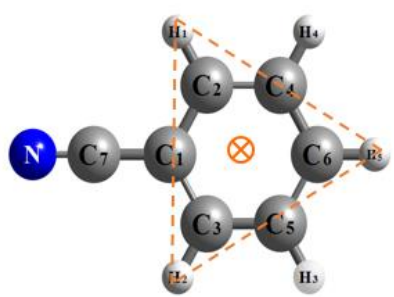

V2

Figure 18. The two hypothetical plates pass through triangle $\mathrm{C} 7-\mathrm{H} 3-\mathrm{H} 4$ atoms and triangle $\mathrm{H} 5-\mathrm{H} 1-\mathrm{H} 2$ atoms. The vectors V1 and V2 pointing outwards perpendicular to each plate are marked with $\otimes$.

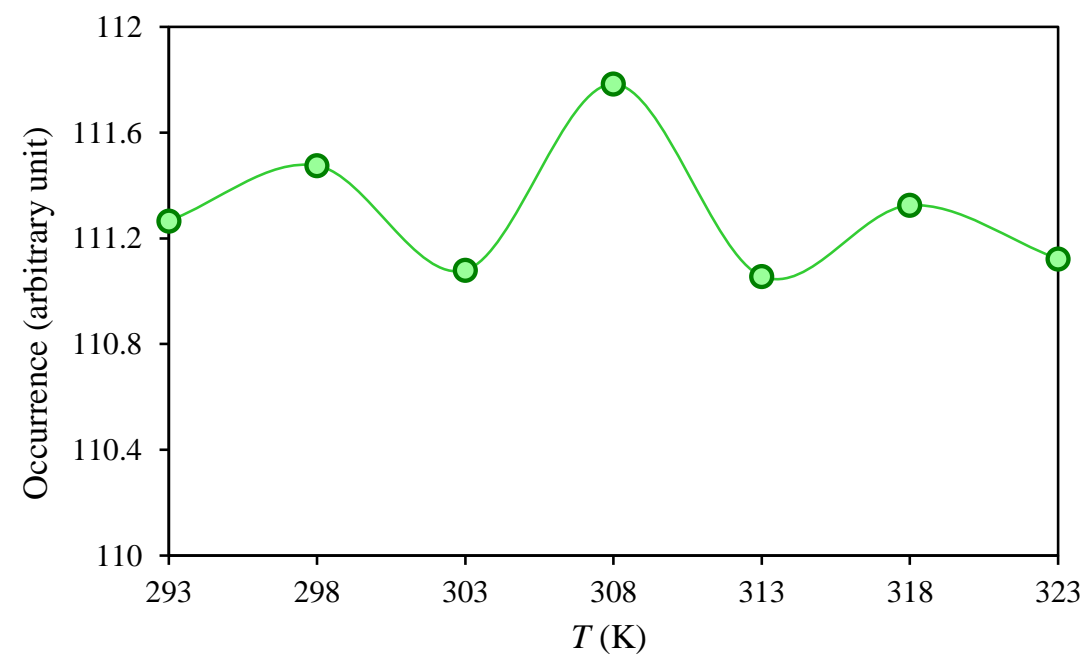

Figure 19. The simulated angular distribution function between the vectors perpendicular to the hypothetical planes specified in Figure 18 at 293-323 K. 
According to Figure 19, the system at $303 \mathrm{~K}$ is just about to make a phase transition from solid (crystal) phase to nematic phases, ${ }^{25-28}$ as having been known very often for liquid crystals. Before this transition, the system is quite ordered, and in the $303-313 \mathrm{~K}$ range, the molecules' planes are aligned preferentially along the shear direction and the liquid is positionally disordered but orientationally ordered. Therefore, part of these molecules contributes more to the viscous isotropic phase and the increase of temperature gives rise to an increasing portion of the isotropic phase at the expense of the anisotropic phase. Above this temperature range, the system becomes disordered again and the isotropic liquid phase prevails. Such behavior would be influential on the physical properties of the system, such as self-diffusion coefficient, viscosity, ... (Figure 9 and 10). As shown in Figure 10, in the temperature range 303 to $313 \mathrm{~K}$, the viscosity is steady. Such behavior can be more severely observed in liquid crystals. ${ }^{43}$

\section{B. Interfacial Properties}

3.B.1. Surface Tension. Molecular dynamics simulation could be used as a prevailing tool in the prediction of surface properties of organic liquids. The surface tension, $\gamma$, is proportional to the integral of the difference between the normal components of the pressure tensor:

$\gamma=-b_{z}\left(P_{x x}+P_{y y}-2 P_{z z}\right) / 4$

where $b_{z}$ is the length of the simulation box along the box principle axis (the $z$-axis) perpendicular to the liquid/vapor interface, and $P_{x x}, P_{y y}$, and $P_{z z}$ are the principal components of the pressure tensor. The result of surface tension simulations (for $20 \mathrm{~ns}$ in NVT ensemble) of liquid BZN at $298 \mathrm{~K}$ is $38.35 \mathrm{mN} / \mathrm{m}$, being in good agreement with the experimental data $(38.33 \mathrm{mN} / \mathrm{m})^{44}$ within $0.1 \mathrm{mN} / \mathrm{m}$. Such a high level of agreement assures the accuracies of the refined force field parameters and allows moving forward studying the surface structure of the liquid benzonitrile. 
Knowledge of the nature and molecular structure of the liquid/vapor interface is of prime interest in surface science applications like the assessment of the transport of chemical species across the interface. The molecular structure and geometry of the liquid system have the basic role in molecular orientation at the liquid surface, reminiscent of the state of minimum mechanical surface stress. The composition of a liquid surface is different from the bulk due to unbalanced forces exerted on particles in this region, which requires the surface to contract to attain a state of minimum stress. The surface molecular structure also determines the liquid surface free energy, a factor that is involved in modeling the theory of solubility. Accordingly, the solubility parameter is ordered by the interfacial tension of the solute and solvent in addition to the solute-solvent interaction. ${ }^{45,46}$ So, the simulation of highly accurate surface properties of liquid BZN are enabling the theoretical study of features of an important solvent in organic reaction synthesis.

3.B.2. Atom's Density Profiles at Interface. MD simulation can be used exclusively to produce spatial positioning and the orientational ordering of molecules at the liquid surface. The molecular density in the liquid bulk and at the interface is rather uniform, while the density of each constituting atom shows specific periodic oscillations characteristics of the atom's environment in the molecules. The density profiles of benzonitrile atoms at $298 \mathrm{~K}$ are shown in Figure 20(a). (see Figure 20(b) for the details at interface). 

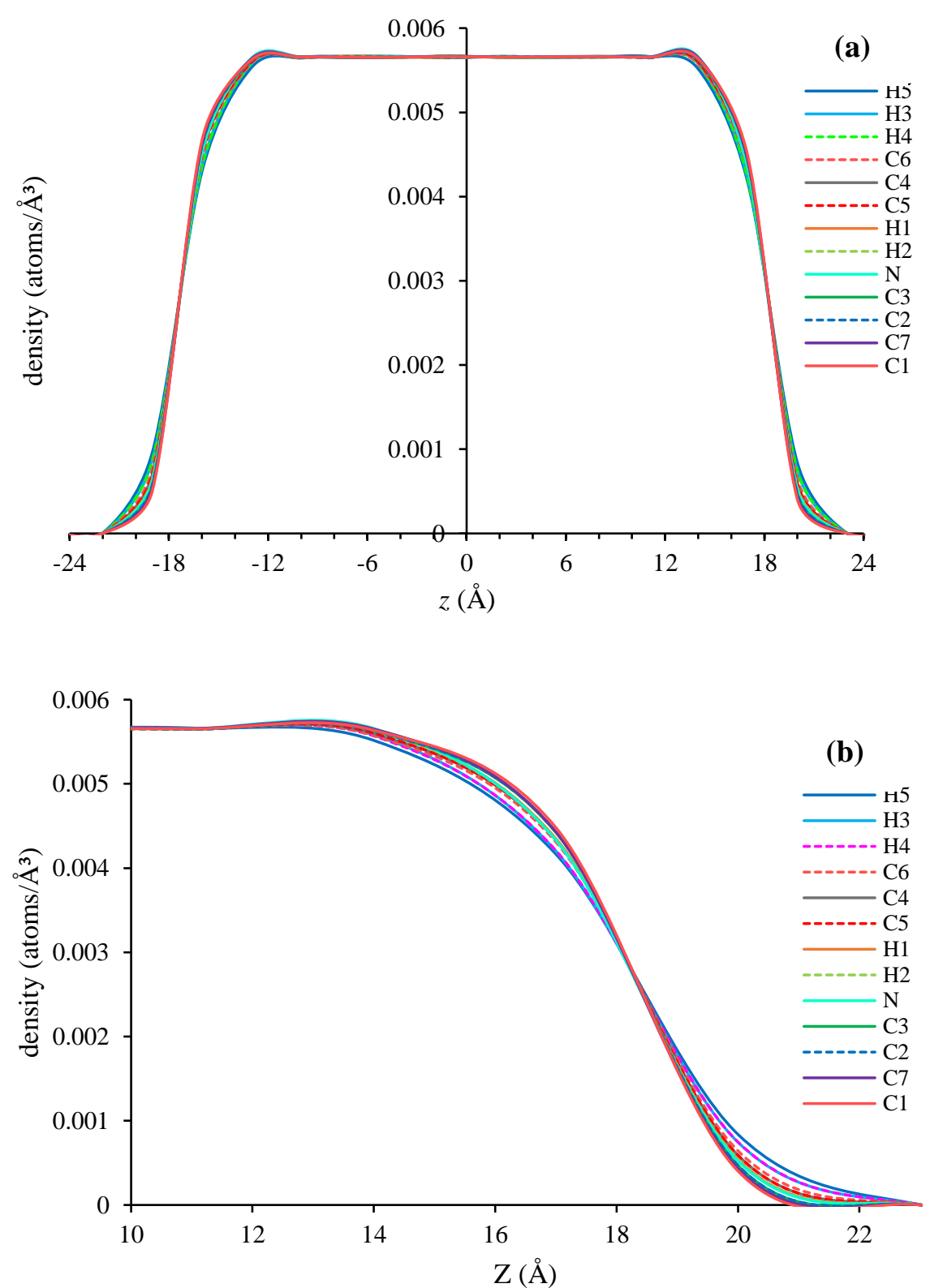

Figure 20. (a) Atom's density profiles of equilibrated benzonitrile liquid/vapor at 298 K. (b) Details of atom's density profiles of equilibrated benzonitrile liquid/vapor at $298 \mathrm{~K}$. 
To analyze the molecular orientation of benzonitrile in the surface region, the angular distribution function (ADF) was calculated by Travis software. ${ }^{38}$ Accordingly, the vector $r_{\mathrm{H}_{5} \cdots \mathrm{N}}$ and the angle $\theta$ (the angle between the vector $r_{\mathrm{H}_{5} \cdots \mathrm{N}}$ and the box principle axis (the $z-$ axis), is defined and used to calculate the molecular orientation during the simulation. The most probable orientations of benzonitrile molecules calculated within the range of $10 \AA$ from the surface indicate that the $\mathrm{H}_{5} \rightarrow \mathrm{N}$ vector makes an angle of $157.5^{\circ}$ with respect to the $\mathrm{z}$-axis. So, the BZN molecule in the surface region is tilted such that the $-\mathrm{C} \equiv \mathrm{N}$ head group is submerged and the $\mathrm{H}_{5}$ tail inclined to air. These results are in good agreement with the results obtained from the density profile.

\section{CONCLUSIONS}

MD simulations remarkably have enabled us to determine the details of bulk liquid benzonitrile structural properties at equilibrium and utilize them to enlighten microscopically the specific phenomenon in non-equilibrium properties such as temperature-dependent of viscosity and diffusion. After elucidating the liquid BZN cluster formation, ${ }^{11}$ and now paying attention to the details of its molecular stacking phenomenon, we could have deconvoluted and extracted the distribution of twist orientational distribution in stacking mode by appropriate implementation of the angle-dependent pair correlation function. Two BZN molecules prefer interacting in stacking mode with their principle molecular axis angled by about $180^{\circ}$ (i.e., twist angle). This firm relative structure is somewhat relaxed for a third BZN molecule admitted to finding a correlation by stacking on, while it is freer to map a wider twist distribution. The Analysis of resulted simulation at different temperatures (within 298-323 K) suggests that the specific singularity in the trend of the temperature-dependent-viscosity centered at about 313 $\mathrm{K}$ can adhere to the twisting of the third molecule with respect to the first one.

\section{AUTHOR INFORMATION}




\section{Corresponding Author}

Mohammad Hadi Ghatee - Department of Chemistry, Shiraz University, Shiraz 71946, Iran; orcid.org/0000-0001-6567-9091; Phone: +98 713 613 7174; Fax: +98 713 6460788Email; mhghatee@shirazu.ac.ir, mhghatee2@gmail.com

\section{Other Authors}

Leila Sakhtemanian - Department of Chemistry, Shiraz University, Shiraz 71946, Iran

\section{Notes}

The authors declare no competing financial interest.

\section{Authorship Contribution Statement}

Leila Sakhtemanian: Investigation, Methodology, Software, Data curation, Visualization, Writing - original draft.

Mohammad Hadi Ghatee: Conceptualization, Methodology, Development for new analysis, Validation, Writing - review \& editing.

\section{ASSOCIATED CONTENT}

Supporting Information. The Supporting Information is available free of charge at: Force field parameters and list of simulation box sizes

\section{ACKNOWLEDGEMENTS}

The authors are indebted to the research council of Shiraz University for financial supports. Computer time is partly provided by the High-Performance Computing research laboratory of the Institute for Research in Fundamental Sciences (IPM).

\section{REFERENCES}

(1) Jia, Q.; Wang, J., N-Heterocyclic Carbene-Catalyzed Convenient Benzonitrile Assembly. Org. Lett. 2016, 18 (9), 2212-2215.

(2) Chatterjee, K.; Dopfer, O., Infrared Signatures of Protonated Benzonitrile. Astrophys. J. 2018, 865 (2), 1-15. 
(3) McGuire, B. A.; Burkhardt, A. M.; Kalenskii, S.; C. N.; Shingledecker, Remijan, A. J.; Herbst, E.; McCarthy. M. C., Detection of the Aromatic Molecule Benzonitrile (c-C6H5CN) in the Interstellar Medium. Science. 2018, 359, 202-205.

(4) Moldoveanu, S. C., Chapter 19 Pyrolysis of Various Derivatives of Carboxylic Acids. Pyrolysis of Organic Molecules. 2010, 28, 579-627.

(5) Li, Z.; Wang, T.; Qi, X.; Yang, Q.; Gao, L.; Zhang, D.; Zhao, X.; Wang, Y., Green Synthesis of Benzonitrile Using Ionic Liquid with Multiple Roles as the Recycling Agent. RSC Adv. 2019, 9, 17631-17638.

(6) Liu, A. A.; Huang, Z.; Deng, G. H.; Guo, Y., Adsorption of Benzonitrile at the Air/Water Interface Studied by Sum Frequency Generation Spectroscopy. Chin Sci Bull, 2013, 58, 15291535 .

(7) Kadish, K. M.; Anderson, J. E., Purification of Solvents for Electroanalysis, Benzonitrile, Dichloromethane, 1, 1-dichloromethane and 1, 2-dichloroethane. Pure \& Appl. Chem. 1987, 59 (5), 703-714.

(8) Larson, R. C.; Iwamoto, R. T., Solvent Effects on the Polarographic Reduction of Metal Ions. I. Benzonitrile-Acetonitrile. J. Am. Chem. Soc. 1960, 82 (13), 3239-3244.

(9) Wright, D.; Caldwell, R.; Moxely, C.; EI-Shall, M. S., Homogeneous Nucleation in Supersaturated Vapors of Polar Molecules: Acetonitrile, Benzonitrile, Nitromethane, and Nitrobenzene. J. Chern. Phys. 1993, 98 (4), 3356-3368.

(10) Yamamoto, R.; Ebata, T.; Mikami, N., Mode dependent Intracluster Vibrational Energy Redistribution Rate in Size-Selected Benzonitrile- $\left(\mathrm{CHCl}_{3}\right) n=1-3$ Clusters. J. Chem. Phys. 2001, 114 (18), 7866-7876.

(11) Sakhtemanian, L.; Ghatee, M. H. Multi-structural Feasibility in Benzonitrile Solvent Through the Multi Hot-potential Centers. J. Mol. Liq. 2021, 336, 116309.

(12) Chang, Y. J.; Castner, E. W., Intermolecular Dynamics of Substituted Benzene and Cyclohexane Liquids, Studied by Femtosecond Nonlinear-Optical Polarization Spectroscopy. J. Phys. Chem. A. 1996, 100, 3330-3343.

(13) Lide, D. R., CRC Handbook of Chemistry and Physics, $84^{\text {th }}$ Edition, CRC Press LLC, 2003-2004.

(14) Borst, D. R.; Pratt, D. W.; Schäfer, M., Molecular Recognition in the Gas Phase. DipoleBound Complexes of Benzonitrile with Water, Ammonia, Methanol, Acetonitrile, and Benzonitrile Itself. Phys. Chem. Chem. Phys. 2007, 9, 4563-4571.

(15) Raghuvansh, P.; Srivastava, S. K.; Singh, R. K.; Asthana, B. P.; Kiefer, W., Hydrogenbonding and Self Association Investigated in the Binary Mixture $\left(\mathrm{C}_{6} \mathrm{H}_{5} \mathrm{C} \equiv \mathrm{N}+\mathrm{CH}_{3} \mathrm{OH}\right)$ via Concentration Dependent Raman Study of the $\mathrm{C} \equiv \mathrm{N}$ Stretching Mode of Benzonitrile $\left(\mathrm{C}_{6} \mathrm{H}_{5} \mathrm{C} \equiv \mathrm{N}\right)$ and ab-initio Calculations. Phys. Chem. Chem. Phys. 2004, 6, 531-536.

(16) Maeyama, T.; Yagi, I.; Murota, Y.; Fujii, A.; Mikami, N., Infrared Vibrational Autodetachment Spectroscopy of Microsolvated Benzonitrile Radical Anions. J. Phys. Chem. A. 2006, 110, 13712-13716. 
(17) Boo, D. W.; Kim, K.; Kim, M. S., Raman Spectroscopy Study of Benzonitrile on Silver Surface. Bull. Korean. Chem. Soc. 1987, 8(4), 251-254.

(18) Nakayama, T.; Inamura, K.; Inoue, Y.; Ikeda, S.; Kishi, K., Adsorption of Benzonitrile and Alkyl Cyanides on Evaporation Nickel and Palladium Films Studied by XPS. Surf. Sci. 1987, 179, 47-58.

(19) Mrozek, M. F.; Wasileski, S. A.; Weaver, M. J., Periodic Trends in ElectrodeChemisorbate Bonding: Benzonitrile on Platinum-Group and Other Noble Metals As Probed by Surface-Enhanced Raman Spectroscopy Combined with Density Functional Theory. J. Am. Chem. Soc. 2001, 123, 12817-12825.

(20) Ateşin, T. A.; Li, T.; Lachaize, S.; García, J. J.; Jones, W. D., Experimental and Theoretical Examination of C-CN Bond Activation of Benzonitrile Using Zerovalent Nickel. Organometallics 2008, 27, 3811-3817.

(21) Okuno, Y.; Yokoyama, T.; Yokoyama, S; Kamikado, T.; Mashiko, S., Theoretical Study of Benzonitrile Clusters in the Gas Phase and Their Adsorption onto a Au(111) Surface. J. Am. Chem. Soc. 2002, 124, 7218-7225.

(22) Aguiar, A. L.; Fagan, S. B.; da Silva, L. B.; Mendes Filho, J.; Souza Filho, A. G., Benzonitrile Adsorption on Fe-Doped Carbon Nanostructures. J. Phys. Chem. C. 2010, 114, 10790-10795.

(23) Whittenburg, S. L.; Wang, C. H., Light Scattering Studies of Transverse Sound Wave and Molecular Motion in Benzonitrile. J. Chem. Phys. 1977, 66, 4995.

(24) Sha, M.; Yamada, S. A.; Fayer, F. D., Orientational Pair Correlations and Local Structure of Benzonitrile from Molecular Dynamics Simulations with Comparisons to Experiments. $J$. Phys. Chem. B. 2021, 125, 3163-3177.

(25) Sidky, H.; Whitmer, J. K., The Emergent Nematic Phase in Ionic Chromonic Liquid Crystals. J. Phys. Chem. B. 2017, 121, 27, 6691-6698.

(26) Kumar, M.; Kumar, S., Liquid Crystals in Photovoltaics: A New Generation of Organic Photovoltaics. Polymer. 2017, 49, 85-111.

(27) Prehm, M.; Liu, F.; Zeng, X.; Ungar, G.; Tschierske, K., Axial-Bundle Phases - New Modes of 2D, 3D, and Helical Columnar Self-Assembly in Liquid Crystalline Phases of Bolaamphiphiles with Swallow Tail Lateral Chains. J. Am. Chem. Soc. 2011, 133, 4906-4916.

(28) Aleksandriiskaya, E. V.; Kuvshinova, S. A.; Novikov, I. V.; Aleksandriiskii, V. V.; Tararykina, T. V.; Maizlish, V. E.; Burmistrov, V. A., Effects of Nonmesomorphic Substituted Benzonitriles on the Dielectric Properties of Cyanobiphenyl Liquid Crystals. Russ. J. Phys. Chem. A. 2008, 82, 1211-1214.

(29) Thote, A. J.; Gupta, R. B., Hydrogen-Bonding Effects in Liquid Crystals for Application to LCDs. Ind. Eng. Chem. Res. 2003, 42, 1129-1136. 
(30) Frisch, M. J.; Trucks, G. W.; Schlegel, H. B.; Scuseria, G. E; Robb, M. A.; Cheeseman, J. R.; Montgomery Jr., J. A.; Vreven, T.; Kudin, K. N.; Burant, J. C.; Millam, J. M.; Iyengar, S. S.; Tomasi, J.; Barone, V.; Mennucci, B.; Cossi, M.; Scalmani, G.; Rega, N.; Petersson, G. A.; Nakatsuji, H.; Hada, M.; Ehara, M.; Toyota, K.; Fukuda, R.; Hasegawa, J.; Ishida, M.; Nakajima, T.; Honda, Y.; Kitao, O.; Nakai, H.; Klene, M.; Li, X.; Knox, J. E.; Hratchian, H. P.; Cross, J. B.; Bakken, V.; Adamo, C.; Jaramillo, J.; Gomperts, R.; Stratmann, R. E.; Yazyev, O.; Austin, A. J.; Cammi, R.; Pomelli, C.; Ochterski, J.; Ayala, P. Y.; Morokuma, K.; Voth, G. A.; Salvador, P.; Dannenberg, J. J.; Zakrzewski, V. G.; Dapprich, S.; Daniels, A. D.; Strain, M. C.; Farkas, O.; Malick, D. K.; Rabuck, A. D.; Raghavachari, K.; Foresman, J. B.; Ortiz, J. V.; Cui, Q.; Baboul, A. G.; Clifford, S.; Cioslowski, J.; Stefanov, B. B.; Liu, G.; Liashenko, A.; Piskorz, P.; Komaromi, I.; Martin, R. L.; Fox, D. J.; Keith, T.; Al-Laham, M. A.; Peng, C. Y.; Nanayakkara, A.; Challacombe, M.; Gill, P. M. W.; Johnson, B. G.; Chen, W.; Wong, M. W.; Gonzalez, C.; Pople, J. A., GAUSSIAN 09 Revision A.02, Gaussian, Inc., Wallingford, CT, 2009.

(31) Breneman, C. M.; Wiberg, K. B. Determining Atom-Centered Monopoles from Molecular Electrostatic Potentials. The Need for High Sampling Density in Formamide Conformational Analysis. J. Comput. Chem. 1990, 11 (3), 361-373.

(32) Smith, W.; Forester, T.; Todorov, I., The DL_POLY Molecular Simulation Package, v.2.18; Daresbury Laboratory: UK (2007).

(33) Jorgensen, W. L.; Tirado-Rives, J., The OPLS [Optimized Potentials for Liquid Simulations] Potential Functions for Proteins, Energy Minimizations for Crystals of Cyclic Peptides and Crambin. J. Am. Chem. Soc. 1988, 110, 1657-1666.

(34) Price, M. L. P.; Ostrovsky, D.; Jorgensen, W. L., Gas-Phase and Liquid-State Properties of Esters, Nitriles, and Nitro Compounds with the OPLS-AA Force Field. J. Comput. Chem. 2001, 22, 1340-1352.

(35) Jorgensen, W. L.; Severance, D. L., Aromatic-Aromatic Interactions: Free Energy Profiles for the Benzene Dimer in Water, Chloroform, and Liquid Benzene. J. Am. Chem. SOC. 1990, $112,4168-4114$.

(36) Nikam, P. S.; Kharat, S. J., Excess Molar Volumes and Deviations in Viscosity of Binary Mixtures of N,N-Dimethylformamide with Aniline and Benzonitrile at (298.15, 303.15, 308.15, and 313.15) K. J. Chem. Eng. Data. 2003, 48, 972-976.

(37) Lei, Y.; Chen, Z.; An, X.; Huang, M.; Shen, W., Measurements of Density and Heat Capacity for Binary Mixtures $\{x$ Benzonitrile $+(1-x)$ (Octane or Nonane $)\} . J$. Chem. Eng. Data. 2010, 55, 4154-4161.

(38) Brehm, M.; Kirchner, B., TRAVIS-a Free Analyzer and Visualizer for Monte Carlo and Molecular Dynamics Trajectories, J. Am. Chem. Soc. 2011, 51, 2007-2023.

(39) Zhao, R.; Zhang, R. Q., A new insight into $\pi-\pi$ stacking involving remarkable orbital interactions. Phys. Chem. Chem. Phys. 2016, 18, 25452-25457.

(40) Hyperchem, Release 7.0, Hypercube Inc. 1115 NW 4th Street, Gainesville, FL 32601 USA. 
(41) Ghatee, M. H.; Moosavi, F., Physisorption of Hydrophobic and Hydrophilic 1-Alkyl-3methylimidazolium Ionic Liquids on the Graphenes. J. Phys. Chem. C. 2011, 115, 5626.

(42) Dixon, A. R.; Khuseynov, D.; Sanov, A. Benzonitrile: Electron Affinity, Excited States, and Anion Aolvation. J. Chem. Phys., 2015, 143, 134306.

(43) Fan, Y.; Dai, Sh.; Tanner, R. I. Rheological Properties of Some Thermotropic Liquid Crystalline Polymers. Korea Aust. Rheol. J. 2003, 15(3), 109-115.

(44) Shukla, R. K.; Kumar, A.; Srivastava, U.; Awasthi, N.; Pandey, J.D., Estimation of the Surface Tensions of Benzonitrile, Chlorobenzene, Benzyl Chloride and Benzyl Alcohol in Mixtures with Benzene by Associated and Non-associated Processes at 298.15, 303.15 and 313.15 K. J Solution Chem. 2012, 41, 1112-1132.

(45) Ghatee, M. H.; Zolghadr, A. R. Surface Tension Measurements of Imidazolium-based Ionic Liquids at Liquid-vapor Equilibrium. Fluid Phase Equilibria. 2008, 263, 168-175.

(46) Ghatee, M. H.; Ghazipour, H. Highly Accurate Liquid-liquid Interfacial Tension Measurement by a Convenient Capillary Apparatus. Fluid Phase Equilibria. 2014, 377, 76-81. 